\title{
PREPROCESSING SPARSE SEMIDEFINITE PROGRAMS \\ VIA MATRIX COMPLETION
}

\author{
By
}

\section{Katsuki Fujisawa \\ Mituhiro Fukuda}

and

Kazuhide Nakata

IMA Preprint Series \# 1969

(March 2004)

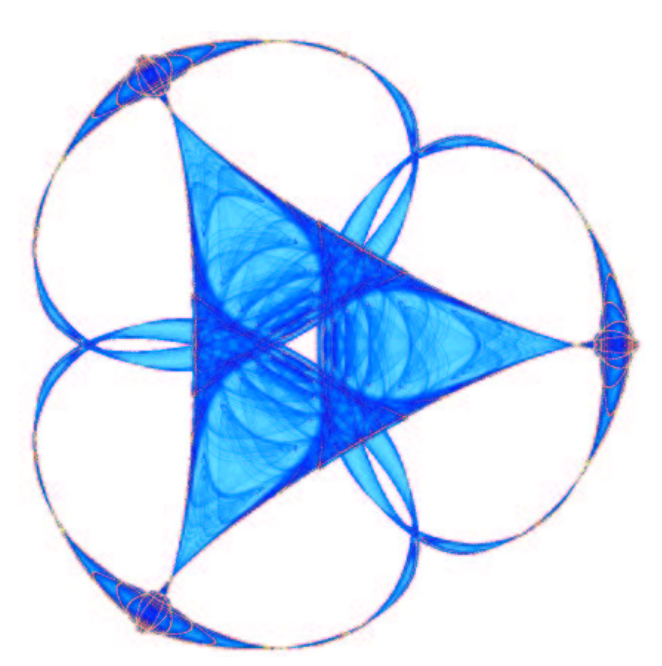

INSTITUTE FOR MATHEMATICS AND ITS APPLICATIONS

UNIVERSITY OF MINNESOTA

514 Vincent Hall

206 Church Street S.E.

Minneapolis, Minnesota 55455-0436

Phone: 612/624-6066 Fax: 612/626-7370

URL: http://www.ima.umn.edu 


\title{
Preprocessing sparse semidefinite programs via matrix completion*
}

\author{
Katsuki Fujisawa (fujisawa@r.dendai.ac.jp) \\ Department of Mathematical Sciences, Tokyo Denki University, \\ Ishizaka, Hatoyama, Saitama, 350-0394, Japan \\ Mituhiro Fukuda (mituhiro@cims.nyu.edu) \\ Department of Mathematics, \\ Courant Institute of Mathematical Sciences, New York University, \\ 251 Mercer Street, New York, NY, 10012-1185 \\ Kazuhide Nakata (knakata@me.titech.ac.jp)
}

The Department of Industrial Engineering and Management, Tokyo Institute of Technology,

2-12-1 Oh-okayama, Meguro, Tokyo, 152-8552, Japan

March, 2004

\begin{abstract}
Considering that preprocessing is an important phase in linear programming, it should be systematically more incorporated in semidefinite programming solvers. The conversion method proposed by the authors (SIAM Journal on Optimization, vol. 11, pp. 647-674, 2000, and Mathematical Programming, Series B, vol. 95, pp. 303-327, 2003) is a preprocessing of sparse semidefinite programs based on matrix completion. This article proposed a new version of the conversion method which employs a flop estimation function inside its heuristic procedure. Extensive numerical experiments are included showing the advantage of preprocessing by the conversion method for very sparse semidefinite programs of certain classes.
\end{abstract}

Keywords: semidefinite programming, preprocessing, sparsity, matrix completion, clique tree, numerical experiments

\footnotetext{
${ }^{*}$ This manuscript was also issued as Research Report B-401, Department of Mathematical and Computing Sciences, Tokyo Institute of Technology, 2-12-1 Oh-okayama Meguro, Tokyo, 152-8552, Japan, March 2004.
} 


\section{Introduction}

Recently, Semidefinite Programming (SDP) has gained attention in several new fronts like in global optimization of problems involving polynomials $[13,14,18]$ and in quantum chemistry [27] besides the well-known applications in system and control theory, in relaxation of combinatorial optimization problems, etc.

These new classes of SDPs characterize as being of large-scale and most of the time as challenging as even to load the problem data onto the physical memory of the computer. As a practical compromise, we often restrict ourselves to solve sparse instances of these large-scale SDPs.

Keeping in mind the above necessity to solve challenging SDPs, this article pushes further the preprocessing procedure named conversion method proposed in $[8,16]$. The conversion method explores the structural sparsity of SDP data matrices, converting a given SDP into an equivalent SDP based on the matrix completion theory. If the SDP data matrices are very sparse, which means that matrix sizes are large at least, the conversion method produces an SDP which can be solved faster and requires less memory than the original SDP when solved by the primal-dual interior-point method [16].

The conversion method is an initial step to consider seriously the preprocessing phase of sparse SDPs as it is common in linear programming [1].

In this sense, we already proposed a general linear transformation which can enhance the sparsity of an SDP [8, Section 6]. Gatermann and Parrilo address another algebraic transformation that can be interpreted as a preprocessing of SDPs under special conditions which can transform the problems into block diagonal SDPs [9]. Also, Toh recognizes the importance of analyzing the data matrices to remove redundant constraints which can cause degeneracy [22]. All of above procedures can be used for sparse and even for dense SDPs.

We strong believe that further investigations are necessary to propose efficient preprocessing to solve large-scale, (sparse) and general SDPs.

The main idea of the conversion method is as follows.

Let $\mathcal{S}^{n}$ denote the space of $n \times n$ symmetric matrices with the Frobenius inner product $\boldsymbol{X} \bullet \boldsymbol{Y}=\sum_{i=1}^{n} \sum_{j=1}^{n} X_{i j} Y_{i j}$ for $\boldsymbol{X}, \boldsymbol{Y} \in \mathcal{S}^{n}$, and $\mathcal{S}_{+}^{n}$ the subspace of $n \times n$ symmetric positive semidefinite matrices. Given $\boldsymbol{A}_{p} \in \mathcal{S}^{n}(p=0,1, \ldots, m)$ and $\boldsymbol{b} \in \mathbb{R}^{m}$, we define the standard equality form $S D P$ by

$$
\begin{cases}\text { minimize } & \boldsymbol{A}_{0} \bullet \boldsymbol{X} \\ \text { subject to } & \boldsymbol{A}_{p} \bullet \boldsymbol{X}=b_{p}(p=1,2, \ldots, m) \\ & \boldsymbol{X} \in \mathcal{S}_{+}^{n},\end{cases}
$$

and its dual by

$$
\begin{cases}\text { maximize } & \sum_{p=1}^{m} b_{p} y_{p} \\ \text { subject to } & \sum_{p=1}^{m} \boldsymbol{A}_{p} y_{p}+\boldsymbol{S}=\boldsymbol{A}_{0} \\ & \boldsymbol{S} \in \mathcal{S}_{+}^{n} .\end{cases}
$$

In this article, we are mostly interested in solving sparse SDPs where the data matrices 
$\boldsymbol{A}_{p}(p=0,1, \ldots, m)$ are sparse, and the dual matrix variable $\boldsymbol{S}=\boldsymbol{A}_{0}-\sum_{p=1}^{m} \boldsymbol{A}_{p} y_{p}$ inherits the sparsity of $\boldsymbol{A}_{p}$ 's.

The sparse structure of an SDP can be represented by the aggregate sparsity pattern of data matrices (alternatively called aggregate density pattern in [5]):

$$
E=\left\{(i, j) \in V \times V:\left[\boldsymbol{A}_{p}\right]_{i j} \neq 0 \text { for some } p \in\{0,1, \ldots, m\}\right\} .
$$

Here $V$ denotes the set $\{1,2, \ldots, n\}$ of row/column indices of data matrices $\boldsymbol{A}_{0}, \boldsymbol{A}_{1}, \ldots, \boldsymbol{A}_{m}$, and $\left[\boldsymbol{A}_{p}\right]_{i j}$ denotes the $(i, j)$ th element of $\boldsymbol{A}_{p} \in \mathcal{S}^{n}$. It is also convenient to identify the aggregate sparsity pattern $E$ with the aggregate sparsity pattern matrix $\boldsymbol{A}(E)$ having unspecified nonzero numerical values in $E$ and zero otherwise.

In accordance with the ideas and definitions presented in $[8,16]$, consider a collection of nonempty subsets $C_{1}, C_{2}, \ldots, C_{\ell}$ of $V$ satisfying

(i) $E \subseteq F \equiv \bigcup_{r=1}^{\ell} C_{r} \times C_{r}$;

(ii) Any partial symmetric matrix $\overline{\boldsymbol{X}}$ with specified elements $\bar{X}_{i j} \in \mathbb{R}((i, j) \in F)$ has a positive semidefinite matrix completion (i.e., given any $\bar{X}_{i j} \in \mathbb{R}((i, j) \in F)$, there exists a positive semidefinite $\boldsymbol{X} \in \mathcal{S}^{n}$ such that $\left.X_{i j}=\bar{X}_{i j} \in \mathbb{R}((i, j) \in F)\right)$ if and only if the submatrices $\overline{\boldsymbol{X}}_{C_{r} C_{r}} \in \mathcal{S}_{+}^{C_{r}}(r=1,2, \ldots, \ell)$.

Here $\overline{\boldsymbol{X}}_{C_{r} C_{r}}$ denotes the submatrix of $\overline{\boldsymbol{X}}$ obtained by deleting all rows/columns $i \notin C_{r}$, $\mathcal{S}_{+}^{C_{r}}$ denotes the set of positive semidefinite symmetric matrices with elements specified in $C_{r} \times C_{r}$. We can assume without loss of generality $C_{1}, C_{2}, \ldots, C_{\ell}$ to be maximal sets with respect to set inclusion.

Then, an equivalent formulation of the SDP (1) can be written as follows [16]:

$$
\left\{\begin{array}{lll}
\operatorname{minimize} & \sum_{(i, j) \in F}\left[\boldsymbol{A}_{0}\right]_{i j} X_{i j}^{\hat{r}(i, j)} & \\
\text { subject to } & \sum_{(i, j) \in F}\left[\boldsymbol{A}_{p}\right]_{i j} X_{i j}^{\hat{r}(i, j)}=b_{p} & (p=1,2, \ldots, m), \\
& X_{i j}^{r}=X_{i j}^{s} & \left(\begin{array}{c}
(i, j) \in\left(C_{r} \cap C_{s}\right) \times\left(C_{r} \cap C_{s}\right), \quad i \geq j, \\
\left(C_{r}, C_{s}\right) \in \mathcal{E}, \quad 1 \leq r<s \leq \ell
\end{array}\right), \\
\boldsymbol{X}^{r} \in \mathcal{S}_{+}^{C_{r}} & (r=1,2, \ldots, \ell),
\end{array}\right.
$$

where $\mathcal{E}$ of the clique tree $\mathcal{T}(\mathcal{K}, \mathcal{E})$ is defined in Section 2, and $\hat{r}(i, j)=\min \{r:(i, j) \in$ $\left.C_{r} \times C_{r}\right\}$ is introduced to avoid the addition of repeated terms. If we further introduce a block-diagonal symmetric matrix variable of the form

$$
\boldsymbol{X}^{\prime}=\left(\begin{array}{ccccc}
\boldsymbol{X}^{1} & \boldsymbol{O} & \boldsymbol{O} & \cdots & \boldsymbol{O} \\
\boldsymbol{O} & \boldsymbol{X}^{2} & \boldsymbol{O} & \cdots & \boldsymbol{O} \\
\vdots & \vdots & \vdots & \ddots & \vdots \\
\boldsymbol{O} & \boldsymbol{O} & \boldsymbol{O} & \cdots & \boldsymbol{X}^{\ell}
\end{array}\right)
$$


and appropriately rearrange all data matrices $\boldsymbol{A}_{0}, \boldsymbol{A}_{1}, \ldots, \boldsymbol{A}_{m}$, and the matrices corresponding to the equalities $X_{i j}^{r}=X_{i j}^{s}$ in (3) to have the same block-diagonal structure as $\boldsymbol{X}^{\prime}$, we obtain an equivalent standard equality primal SDP.

Observe that the original standard equality primal SDP (1) has a single matrix variable of size $n \times n$ and $m$ equality constraints. After the conversion, the SDP (3) has

(a) $\ell$ matrices of size $n_{r} \times n_{r}, n_{r} \leq n(r=1,2, \ldots, \ell)$, and

(b) $m_{+}=m+\sum_{\left(C_{r}, C_{s}\right) \in \mathcal{E}} g\left(C_{r} \cap C_{s}\right)$ equality constraints where $g(C)=\frac{|C|(|C|+1)}{2}$,

where $n_{r} \equiv\left|C_{r}\right|$ denotes the number of elements of $C_{r}$.

In this article, we propose a new version of the conversion method which tries to convert a sparse SDP predicting a priori the flops of solving it by the primal-dual interior-point method. The original conversion method $[8,16]$ has a simple heuristic routine based only on the matrix sizes (see Subsection 3.2) which can be deficient in the sense of ignoring the actual computation of the numerical linear algebra in SDP solvers. This work is a tentative of refining it, and a flop estimation function is introduced for this purpose. The flops to compute the Schur Complement Matrix (SCM) [6] and other routines like solving the SCM linear equation, and computing eigenvalues can be roughly estimated as a function of equality constraints $m$, matrix sizes $n$ 's, and data sparsity. The parameters of the newly introduced function are estimated by a simple statistical method based on ANOVA (analysis of variance). Finally, this function is used in a new heuristic routine to generate equivalent SDPs.

The new version of the conversion method is compared with the original version with slight improvement and SDPs without conversion through extensive numerical experiments using SDPA 6.00 [25] and SDPT3 3.02 [23] on selected sparse SDPs from different classes as a tentative of detecting SDPs which are suitable for the conversion method. We can conclude that preprocessing by the conversion method becomes more advantageous as the SDPs are sparse. In particular, it seems that sparse SDPs which have less than $5 \%$ on the extended sparsity pattern (see Sections 2 for its definition) can be solved very efficiently in general. Preprocessing by the conversion method is very advisable for sparse SDPs since we can obtain a speed up of 10 100 times in some cases, and even in the eventual cases when solving the original problem is faster, preprocessed SDPs take at most two times more to solve in most of the cases considered here.

Some other related work that also explores sparsity and matrix completions are the completion method [8, 16], and its parallel version [17]. Also, Burer proposed a primal-dual interior-point method restricted on the space of partial positive definite matrices [5].

The rest of the article is written as follows. Section 2 revised some graph related theory which has a strong connection with matrix completion. Section 3 presents the general framework of the conversion method in a neat way, revises the original version in detail, and proposes a minor modification. Section 4 describes the newly proposed conversion method which estimates the flops of each iteration of primal-dual interior-point method solvers. Finally, Section 5 presents an extensive numerical results comparing the performance of the two conversion methods with SDPs without preprocessing. 


\section{Preliminaries}

The details of this section can be found in $[3,8,16]$ and references therein. Let $G\left(V, E^{\circ}\right)$ denote a graph where $V=\{1,2, \ldots, n\}$ is the vertex set, and $E^{\circ}$ is the edge set defined as $E^{\circ}=E \backslash\{(i, i): i \in V\}, E \subseteq V \times V$. A graph $G\left(V, F^{\circ}\right)$ is called chordal, triangulated or rigid circuit if every cycle of length $\geq 4$ has a chord (an edge connecting two non-consecutive vertices of the cycle).

There is a close connection between chordal graphs and positive semidefinite matrix completions that has been fundamental in the conversion method [8, 16], i.e., (ii) in Introduction holds if and only if the associate graph $G\left(V, F^{\circ}\right)$ of $F$ given in (i) is chordal $[8,10]$. We further observe that amazingly the same fact was proved independently in graphical models in statistics [15], and its known as decomposable models [24].

Henceforth, we assume that $G\left(V, F^{\circ}\right)$ denotes a chordal graph. We call $F$ an extended sparsity pattern of $E$ and $G\left(V, F^{\circ}\right)$ a chordal extension or filled graph of $G\left(V, E^{\circ}\right)$. Notice that obtaining a chordal extension $G\left(V, F^{\circ}\right)$ from $G\left(V, E^{\circ}\right)$ corresponds to adding new edges to $G\left(V, E^{\circ}\right)$ in order to $G\left(V, F^{\circ}\right)$ becomes a chordal graph.

Chordal graphs are well-known structures in graph theory, and can be characterized for instance as follows. A graph is chordal if and only if we can construct a clique tree from it. Although there are several equivalent ways to define clique trees, we employ the following one based on the clique-intersection property (CIP) which will be useful throughout the article.

Let $\mathcal{K}=\left\{C_{1}, C_{2}, \ldots, C_{\ell}\right\}$ be any family of maximal subsets of $V=\{1,2, \ldots, n\}$. Let $\mathcal{T}(\mathcal{K}, \mathcal{E})$ be a tree formed by vertices from $\mathcal{K}$ and edges from $\mathcal{E} \subseteq \mathcal{K} \times \mathcal{K} . \mathcal{T}(\mathcal{K}, \mathcal{E})$ is called a clique tree if it satisfies the clique-intersection property (CIP):

(CIP) For each pair of vertices $C_{r}, C_{s} \in \mathcal{K}$, the set $C_{r} \cap C_{s}$ is contained in every vertex on the (unique) path connecting $C_{r}$ and $C_{s}$ in the tree.

In particular, we can construct a clique tree $\mathcal{T}(\mathcal{K}, \mathcal{E})$ from the chordal extension $G\left(V, F^{\circ}\right)$ if we take $\mathcal{K}=\left\{C_{1}, C_{2}, \ldots, C_{\ell}\right\}$ as the family of all maximal cliques of $G\left(V, F^{\circ}\right)$, and define appropriately the edge set $\mathcal{E} \subseteq \mathcal{K} \times \mathcal{K}$ for $\mathcal{T}(\mathcal{K}, \mathcal{E})$ to satisfy the CIP.

Clique trees can be computed efficiently from a chordal graph. We observe further that clique trees are not uniquely determined for a given chordal graph. Though it is known that the multiset of separators $\left\{C_{r} \cap C_{s}:\left(C_{r}, C_{s}\right) \in \mathcal{E}\right\}$ is invariant for all clique trees $\mathcal{T}(\mathcal{K}, \mathcal{E})$ of a given chordal graph, a fact suitable for our purpose together with the CIP.

The following two lemmas will be very important in the development of the conversion method in the next section. Figure 1 illustrates Lemmas 2.1 and 2.2.

Lemma 2.1 [16] Let $\mathcal{T}(\mathcal{K}, \mathcal{E})$ be a clique tree of $G\left(V, F^{\circ}\right)$, and suppose that $\left(C_{r}, C_{s}\right) \in \mathcal{E}$. We construct a new tree $\mathcal{T}^{\prime}\left(\mathcal{K}^{\prime}, \mathcal{E}^{\prime}\right)$ merging $C_{r}$ and $C_{s}$, i.e., replacing $C_{r}, C_{s} \in \mathcal{K}$ by $C_{r} \cup C_{s} \in$ $\mathcal{K}^{\prime}$, deleting $\left(C_{r}, C_{s}\right) \in \mathcal{E}$, and replacing $\left(C_{r}, C\right),\left(C_{s}, C\right) \in \mathcal{E}$ by $\left(C_{r} \cup C_{s}, C\right) \in \mathcal{E}^{\prime}$. Then $\mathcal{T}^{\prime}\left(\mathcal{K}^{\prime}, \mathcal{E}^{\prime}\right)$ is a clique tree of $G\left(V, F^{\prime \circ}\right)$, where $F^{\prime}=\left\{(i, j) \in C_{r}^{\prime} \times C_{r}^{\prime}: r=1,2, \ldots, \ell^{\prime}\right\}$ for $\mathcal{K}^{\prime}=\left\{C_{1}^{\prime}, C_{2}^{\prime}, \ldots, C_{\ell^{\prime}}^{\prime}\right\}, \ell^{\prime}=\ell-1$. Moreover, let $m_{+}$be defined as in (b) (in Introduction), and $m_{+}^{\prime}$ be the corresponding one for $\mathcal{T}^{\prime}\left(\mathcal{K}^{\prime}, \mathcal{E}^{\prime}\right)$. Then $m_{+}^{\prime}=m_{+}-g\left(C_{r} \cap C_{s}\right)$. 
Lemma 2.2 [16] Let $\mathcal{T}(\mathcal{K}, \mathcal{E})$ be a clique tree of $G\left(V, F^{\circ}\right)$, and suppose that $\left(C_{r}, C_{q}\right),\left(C_{s}, C_{q}\right) \in$ $\mathcal{E}$. We construct a new tree $\mathcal{T}^{\prime}\left(\mathcal{K}^{\prime}, \mathcal{E}^{\prime}\right)$ in the following way:

(i) If $C_{r} \cup C_{s} \nsupseteq C_{q}$, merge $C_{r}$ and $C_{s}$, i.e., replace $C_{r}, C_{s} \in \mathcal{K}$ by $C_{r} \cup C_{s} \in \mathcal{K}^{\prime}$ and replace $\left(C_{r}, C\right),\left(C_{s}, C\right) \in \mathcal{E}$ by $\left(C_{r} \cup C_{s}, C\right) \in \mathcal{E}^{\prime}$

(ii) Otherwise, merge $C_{r}, C_{s}$ and $C_{q}$, i.e., replace $C_{r}, C_{s}, C_{q} \in \mathcal{K}$ by $C_{r} \cup C_{s} \cup C_{q} \in \mathcal{K}^{\prime}$, delete $\left(C_{r}, C_{q}\right),\left(C_{s}, C_{q}\right) \in \mathcal{E}$ and replace $\left(C_{r}, C\right),\left(C_{s}, C\right),\left(C_{q}, C\right) \in \mathcal{E}$ by $\left(C_{r} \cup C_{s} \cup C_{q}, C\right) \in$ $\mathcal{E}^{\prime}$.

Then $\mathcal{T}^{\prime}\left(\mathcal{K}^{\prime}, \mathcal{E}^{\prime}\right)$ is a clique tree of $G\left(V, F^{\prime \circ}\right)$, where $F^{\prime}=\left\{(i, j) \in C_{r}^{\prime} \times C_{r}^{\prime}: r=1,2, \ldots, \ell^{\prime}\right\}$ for $\mathcal{K}^{\prime}=\left\{C_{1}^{\prime}, C_{2}^{\prime}, \ldots, C_{\ell^{\prime}}^{\prime}\right\}, \ell^{\prime}=\ell-1$ (case $i$ ) and $\ell^{\prime}=\ell-2$ (case ii). Moreover, let $m_{+}$be defined as in (b) (in Introduction), and $m_{+}^{\prime}$ be the corresponding one for $\mathcal{T}^{\prime}\left(\mathcal{K}^{\prime}, \mathcal{E}^{\prime}\right)$. Then, we have respectively

(i) $m_{+}^{\prime}=m_{+}-g\left(C_{r} \cap C_{q}\right)-g\left(C_{s} \cap C_{q}\right)+g\left(\left(C_{r} \cup C_{s}\right) \cap C_{q}\right)$ and;

(ii) $m_{+}^{\prime}=m_{+}-g\left(C_{r} \cap C_{q}\right)-g\left(C_{s} \cap C_{q}\right)$.

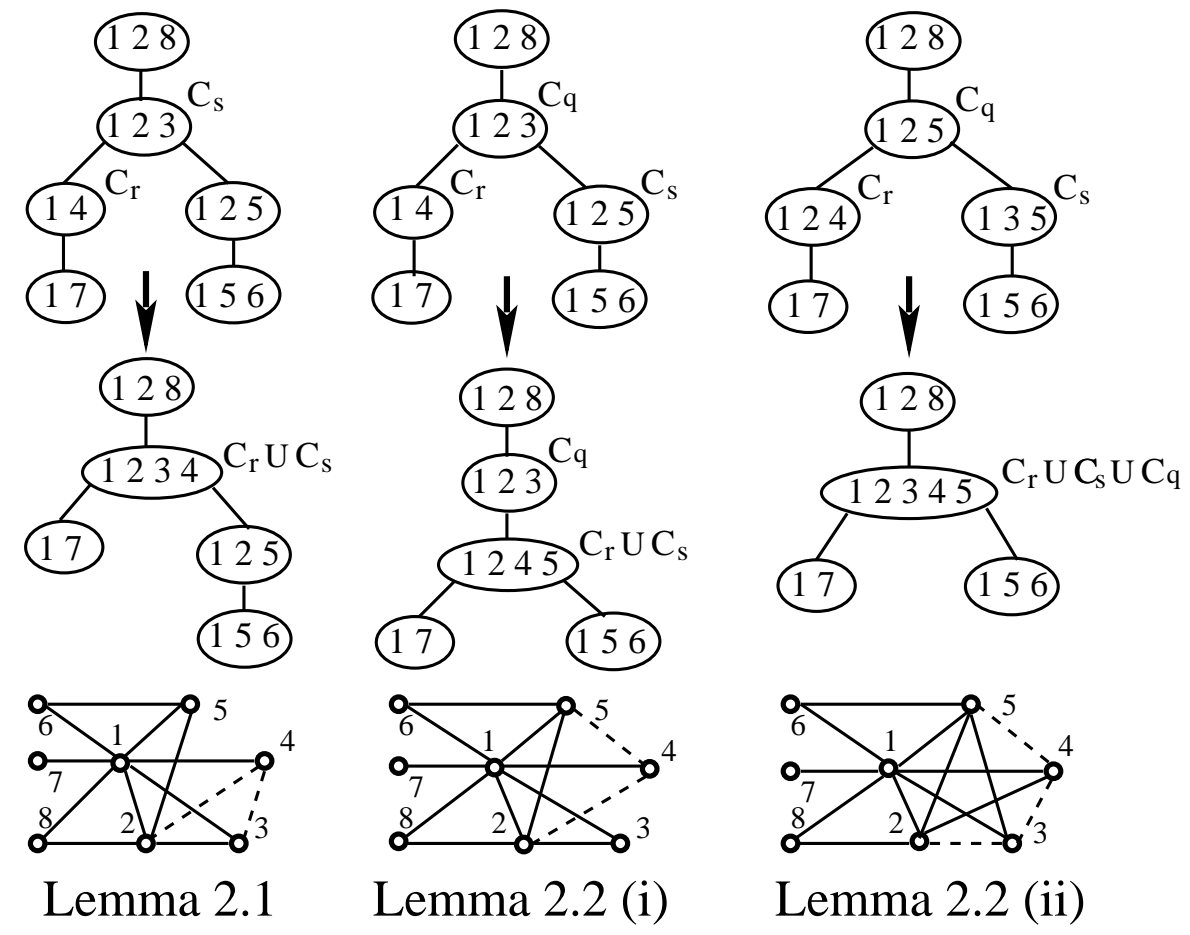

Figure 1: Illustration of Lemmas 2.1 and 2.2. Clique tree $\mathcal{T}(\mathcal{K}, \mathcal{E})$ before the merging (above), clique tree $\mathcal{T}^{\prime}\left(\mathcal{K}^{\prime}, \mathcal{E}^{\prime}\right)$ after the merging (middle), and the associate chordal graph (bottom). Doted lines denote the edges added to the graph because of the clique merging. 


\section{Conversion Method}

\subsection{An Outline}

An implementable conversion method is summarized in Algorithm 3.1. See [16] for details.

Algorithm 3.1 Input: sparse SDP; Output: Equivalent SDP with small block matrices;

Step 1. Read the SDP data and determine the aggregate sparsity pattern E.

Step 2. Find an ordering of rows/columns $V=\{1,2, \ldots, n\}$ which possibly provides lesser fill-in in the aggregate sparsity matrix $\boldsymbol{A}(E)$ (e.g., Spooles 2.2 [2] and METIS 4.0.1 [11]).

Step 3. From the ordering above, perform a symbolic Cholesky factorization onto $\boldsymbol{A}(E)$ associated with $G\left(V, E^{\circ}\right)$ to determine a chordal extension $G\left(V, F^{\circ}\right)$.

Step 4. Compute a clique tree $\mathcal{T}(\mathcal{K}, \mathcal{E})$ from $G\left(V, F^{\circ}\right)$.

Step 5. Use some heuristic procedure to diminish the overlaps $C_{r} \cap C_{s}$ between adjacent cliques, i.e., $\left(C_{r}, C_{s}\right) \in \mathcal{E}$ such that $C_{r}, C_{s} \in \mathcal{K}$, and determine a new clique tree $\mathcal{T}^{*}\left(\mathcal{K}^{*}, \mathcal{E}^{*}\right)$.

Step 6. Convert the original $\operatorname{SDP}$ (1) into (3) using information on $\mathcal{T}^{*}\left(\mathcal{K}^{*}, \mathcal{E}^{*}\right)$.

One of the most important issues of the conversion method is to obtain a suitable chordal extension $G\left(V, F^{\circ}\right)$ of $G\left(V, E^{\circ}\right)$ which allow us to apply a positive semidefinite matrix completion to the original sparse SDP (1).

We also known that a chordal extension $G\left(V, F^{\circ}\right)$ of $G\left(V, E^{\circ}\right)$ can be obtained easily if we perform a symbolic Cholesky factorization on the aggregate sparsity pattern matrix $\boldsymbol{A}(E)$ according to any reordering of $V=\{1,2, \ldots, n\}$. Unfortunately, the problem of finding such an ordering which minimizes the fill-in in $\boldsymbol{A}(E)$ is $\mathcal{N} \mathcal{P}$-complete. Therefore, we rely on some heuristic packages to determine an ordering which possible give less fill-in in Step 2.

Once we have a clique tree $\mathcal{T}(\mathcal{K}, \mathcal{E})$ at Step 4, we can obtain an completely equivalent SDP to the original one with smaller block matrices but with larger number of equality constraints after Step 6. This step consists in visiting once each of the cliques in the clique tree $\mathcal{T}^{*}\left(\mathcal{K}^{*}, \mathcal{E}^{*}\right)$ in order to determine the overlapping elements of $C_{r} \cap C_{s}\left(\left(C_{r}, C_{s}\right) \in\right.$ $\left.\mathcal{E}^{*}, C_{r}, C_{s} \in \mathcal{K}^{*}\right)$. However, as mentioned in Introduction, we obtain at last an SDP with

$\left(\mathrm{a}^{\prime}\right) \ell^{*}=\left|\mathcal{K}^{*}\right|$ matrices of size $n_{r} \times n_{r},\left|C_{r}\right| \equiv n_{r} \leq n\left(r=1,2, \ldots, \ell^{*}\right)$, and

(b') $m_{+}=m+\sum_{\left(C_{r}, C_{s}\right) \in \mathcal{E}^{*}} g\left(C_{r} \cap C_{s}\right)$ equality constraints.

If we opt for an chordal extension $G\left(V, F^{\circ}\right)$ that gives less fill-in as possible at Step 3 (and therefore an ordering at Step 2), we obtain an SDP (3) with $\ell^{*}$ smallest block matrices as possible of size $n_{r}\left(r=1,2, \ldots, \ell^{*}\right)$, and more crucial, a large number of equality constraints $m_{+} \gg m$. One of the keys to obtain a good conversion is to balance the factors (a') and (b') above to have few flop operations as possible when solving by an SDP solver. Therefore, there is a necessity to use a heuristic procedure at Step 5 which manipulates directly the clique trees, which in practice means that we are adding new edges to the chordal graph 
$G\left(V, F^{\circ}\right)$ to create a new chordal graph $G\left(V,\left(F^{*}\right)^{\circ}\right)$ with $F^{*} \supseteq F$ and a corresponding clique tree $\mathcal{T}^{*}\left(\mathcal{K}^{*}, \mathcal{E}^{*}\right)$, which has less overlaps between adjacent cliques.

One can also consider an algorithm that avoids all of these manipulations and finds an ordering at Step 2 which gives the best chordal extension (and a clique tree), and therefore, makes Step 5 unnecessary. However, this alternative seems too beyond of our perspectives due to the complexity of the problem: predicting flops of a sophisticated optimization solver from the structure of the feeding data, and producing the best ordering of rows/columns of the aggregate sparsity matrix $\boldsymbol{A}(E)$.

Therefore, we assume Algorithm 3.1 as a pragmatic strategy for the conversion method.

The details of Step 5 are given in the next subsection.

\subsection{A Simple Heuristic Algorithm to Balance the Sizes of SDPs}

We will make use of Lemmas 2.1 and 2.2 here. These lemmas tell us a sufficient condition for merging the cliques in the clique tree without losing the CIP. Once we merge two cliques $C_{r}$ and $C_{s}$, it will reduce the total number of block matrices by one, the number of equality constraints by $g\left(C_{r} \cap C_{s}\right)$, and increase the size of one of block matrices in (3) in the simplest case (Lemma 2.1). Also, observe that these operations add extra edges to the chordal graph $G\left(V, F^{\circ}\right)$ to produce a chordal graph $G\left(V,\left(F^{*}\right)^{\circ}\right)$ which is associated with the clique tree $\mathcal{T}^{*}\left(\mathcal{K}^{*}, \mathcal{E}^{*}\right)$.

As we have mentioned before, it seems very difficult to find an exact criterion which determines when two maximal cliques $C_{r}$ and $C_{s}$ satisfying the hypothesis of Lemmas 2.1 or 2.2 should be merged or not to balance the factors (a') and (b') in terms of flops. Therefore, we have adopted one simple criterion [16].

Let $\zeta \in(0,1)$. We decide to merge the cliques $C_{r}$ and $C_{s}$ in $\mathcal{T}(\mathcal{K}, \mathcal{E})$ if

$$
h\left(C_{r}, C_{s}\right) \equiv \min \left\{\frac{\left|C_{r} \cap C_{s}\right|}{\left|C_{r}\right|}, \frac{\left|C_{r} \cap C_{s}\right|}{\left|C_{s}\right|}\right\} \geq \zeta .
$$

Although criterion (4) is not complete, it takes into account the sizes of the cliques $C_{r}$ and $C_{s}$ involved, and compares them with the size of common indices $\left|C_{r} \cap C_{s}\right|$. Also, the minimization among the two quantities avoids the merging of a large and a small cliques which share a reasonable number of indices if compared with the smaller one. In particular, this criterion ignores the smallest part between $\left|C_{r}\right|$ and $\left|C_{s}\right|$.

Again, we opt for a specific order to merge the cliques in the clique tree as given in Algorithm 3.2 [16]. Variations are possible but is seems too demanding for our final purpose.

Here we introduce a new parameter $\eta$, which was not considered in the previous version [16], since we think that the number of equality constraints in (3) must diminish considerably when merging cliques to reduce the overall computational time after the conversion.

\footnotetext{
Algorithm 3.2 Diminishing the number of maximal cliques in the clique tree $\mathcal{T}(\mathcal{K}, \mathcal{E})$.
} 
Choose a maximal clique in $\mathcal{K}$ to be the root for $\mathcal{T}(\mathcal{K}, \mathcal{E})$, and let $\zeta, \eta \in(0,1)$

for each maximal clique $C$ which was visited for the last time in $\mathcal{T}(\mathcal{K}, \mathcal{E})$ in a depth-first search

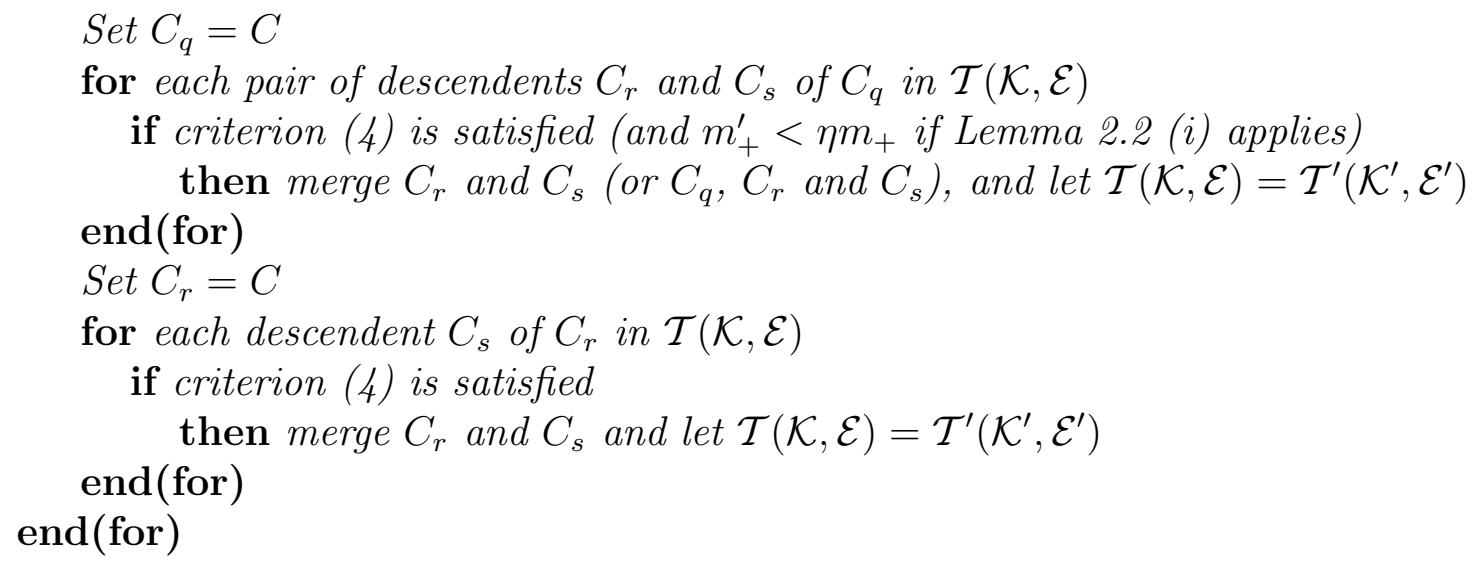

Unfortunately in practice, each of the above parameters depends on the SDP. In Subsection 4.2 we will try to estimate the "best parameter" using a statistical method. The inclusion of the new parameter $\eta$, and the estimation of good values for both parameters $\zeta$ and $\eta$ in (4) makes Algorithm 3.2 an improvement over the previous one [16].

\section{Conversion Method with Flop Estimation}

So far, we have presented the algorithms implemented in [16] with a slight modification. Our proposal here is to replace criterion (4) with a new one which approximately predicts the flops of an SDP solver.

\subsection{Estimating Flops of SDP Codes}

At first, we will restrict our discussion on a particular solver: SDPA 6.00 [25] which is an implementation of the Mehrotra-type primal-dual predictor-corrector interior-point method using the HRVW/KSH/M direction.

The actual flops of sophisticated solvers are very complex and difficult to predict. They depend not only on the sizes and the structure of a particular SDP, but also on the actual data, sparsity, degeneracy of the problem, etc. However, we know a rough estimation of flops per iteration of a primal-dual interior-point method. It consists on the flops to compute the SCM [6], plus other parts which depend on the actual size and eventually on the number of nonzero elements of data matrices of a given SDP which is $\mathcal{O}\left(m^{3}+\sum_{r=1}^{\ell} n_{r}^{3}+m \sum_{r=1}^{\ell} n_{r}^{2}\right)$ [6].

In particular, SDPA 6.00 considers the sparsity of data matrices $\boldsymbol{A}_{p}(p=1,2, \ldots, m)$, and employs the formula $\mathcal{F}_{*}[7]$ to compute the SCM. For each $p=1,2, \ldots, m$ and $r=1,2, \ldots, \ell$, let $f_{p}(r)$ denote the number of nonzero elements of $\boldsymbol{A}_{p}$ for the corresponding block matrix with dimension $n_{r} \times n_{r}$. Analogously, $f_{\Sigma}(r)$ denotes the number of nonzero elements of $\boldsymbol{A}(E)$ for the corresponding block matrix. In the following discussion about flop estimate, 
there will be no loss of generality in considering that $f_{p}(r)(p=1,2, \ldots, m)$ are sorted in non-increasing order for each $r=1,2, \ldots, \ell$ fixed.

Given a constant $\kappa \geq 1$, the cost to compute the SCM is given by

$$
\sum_{r=1}^{\ell} S\left(f_{1}(r), f_{2}(r), \ldots, f_{m}(r), n_{r}\right)
$$

where

$$
\begin{array}{r}
S\left(f_{1}(r), f_{2}(r), \ldots, f_{m}(r), n_{r}\right)=\sum_{p=1}^{m} \min \left\{\kappa n_{r} f_{p}(r)+n_{r}^{3}+\kappa \sum_{q=p}^{m} f_{q}(r),\right. \\
\kappa n_{r} f_{p}(r)+\kappa\left(n_{r}+1\right) \sum_{q=p}^{m} f_{q}(r), \\
\left.\kappa\left(2 \kappa f_{p}(r)+1\right) \sum_{q=p}^{m} f_{q}(r)\right\} .
\end{array}
$$

Considering also the sparsity of block matrices, we propose the following formula for flop estimate of each iteration of the primal-dual interior-point method. Let $\alpha, \beta, \gamma>0$,

$$
\begin{aligned}
& C_{\alpha, \beta, \gamma}\left(f_{1}, f_{2}, \ldots, f_{m}, m, n_{1}, n_{2}, \ldots, n_{\ell}\right) \\
& =\sum_{r=1}^{\ell} S\left(f_{1}(r), f_{2}(r), \ldots, f_{m}(r), n_{r}\right)+\alpha m^{3}+\beta \sum_{r=1}^{\ell} n_{r}^{3}+\gamma \sum_{r=1}^{\ell} n_{r} f_{\Sigma}(r) .
\end{aligned}
$$

Observe that the term $\mathcal{O}\left(m \sum_{r=1}^{\ell} n_{r}^{2}\right)$ was not include in the proposed formula for the reasons explained next.

Our goal is to replace criterion (4) which defines when we should merge the cliques $C_{r}$ and $C_{s}$ in $\mathcal{T}(\mathcal{K}, \mathcal{E})$. Therefore we just need to consider the difference of (6) before and after merging $C_{r}$ and $C_{s}$ to determine if it is advantageous or not to execute this operation (see Step 5 of Algorithm 3.1). Considering the most complicate case Lemma 2.2 (ii), we decide to merge if

$$
\begin{aligned}
& C_{\alpha, \beta, \gamma}^{\text {before }}\left(f_{1}, f_{2}, \ldots, f_{m}, m, n_{1}, n_{2}, \ldots, n_{r}, n_{s}, n_{q}, \ldots, n_{\ell}\right) \\
& -C_{\alpha, \beta, \gamma}^{\text {after }}\left(f_{1}, f_{2}, \ldots, f_{m_{+}}, m_{+}, n_{1}, n_{2}, \ldots, n_{t}, \ldots, n_{\ell^{\prime}}\right) \\
& =S\left(f_{1}(r), f_{2}(r), \ldots, f_{m}(r), n_{r}\right)+S\left(f_{1}(s), f_{2}(s), \ldots, f_{m}(s), n_{s}\right)+S\left(f_{1}(q), f_{2}(q), \ldots, f_{m}(q), n_{q}\right) \\
& -S\left(f_{1}(t), f_{2}(t), \ldots, f_{m_{+}}(t), n_{t}\right)+\alpha\left(m^{3}-m_{+}^{3}\right)+\beta\left(n_{r}^{3}+n_{s}^{3}+n_{q}^{3}-n_{t}^{3}\right) \\
& +\gamma\left(n_{r} f_{\Sigma}(r)+n_{s} f_{\Sigma}(s)+n_{q} f_{\Sigma}(q)-n_{t} f_{\Sigma}(t)\right)>0,
\end{aligned}
$$

where $t$ denotes a new index of a block matrix (clique) after merging $C_{r}, C_{s}$ and $C_{q}, n_{t}=$ $\left|C_{r} \cup C_{s} \cup C_{q}\right|=\left|C_{r} \cup C_{s}\right|$, and $\ell^{\prime}=\ell-2$. Criterion (7) has the advantage of just carrying out the computation of corresponding block matrices (cliques). The inclusion of $\mathcal{O}\left(m \sum_{r=1}^{\ell} n_{r}^{2}\right)$ in (6) would complicate the evaluation of (7) since it would involve information on all block matrices.

Another simplification we imposed in the actual implementation was to replace $f_{\Sigma}(\cdot)$ by $f_{\Sigma}^{\prime}(\cdot)$

$$
f_{\Sigma}\left(n_{t}\right) \geq f_{\Sigma}^{\prime}\left(n_{t}\right) \equiv \max \left\{f_{\Sigma}(r), f_{\Sigma}(s), f_{\Sigma}(r)+f_{\Sigma}(s)-\left|C_{r} \cap C_{s}\right|^{2}\right\}
$$


which avoids us to recalculate the non-zeros elements of each corresponding block matrix at every evaluation of (7). We observe however that $f_{p}(r)\left(p=1,2, \ldots, m_{+}, r=1,2, \ldots, \ell^{\prime}\right)$ can be always retrieved exactly.

The remaining cases Lemma 2.1 and Lemma 2.2 (i) follow analogously.

Preliminary numerical experiments using criterion (7) showed that its computation is still very expensive even after several simplifications. Therefore, we opted to implement Algorithm 4.1 which is similar to Algorithm 3.2 and utilizes a hybrid criterion with (4).

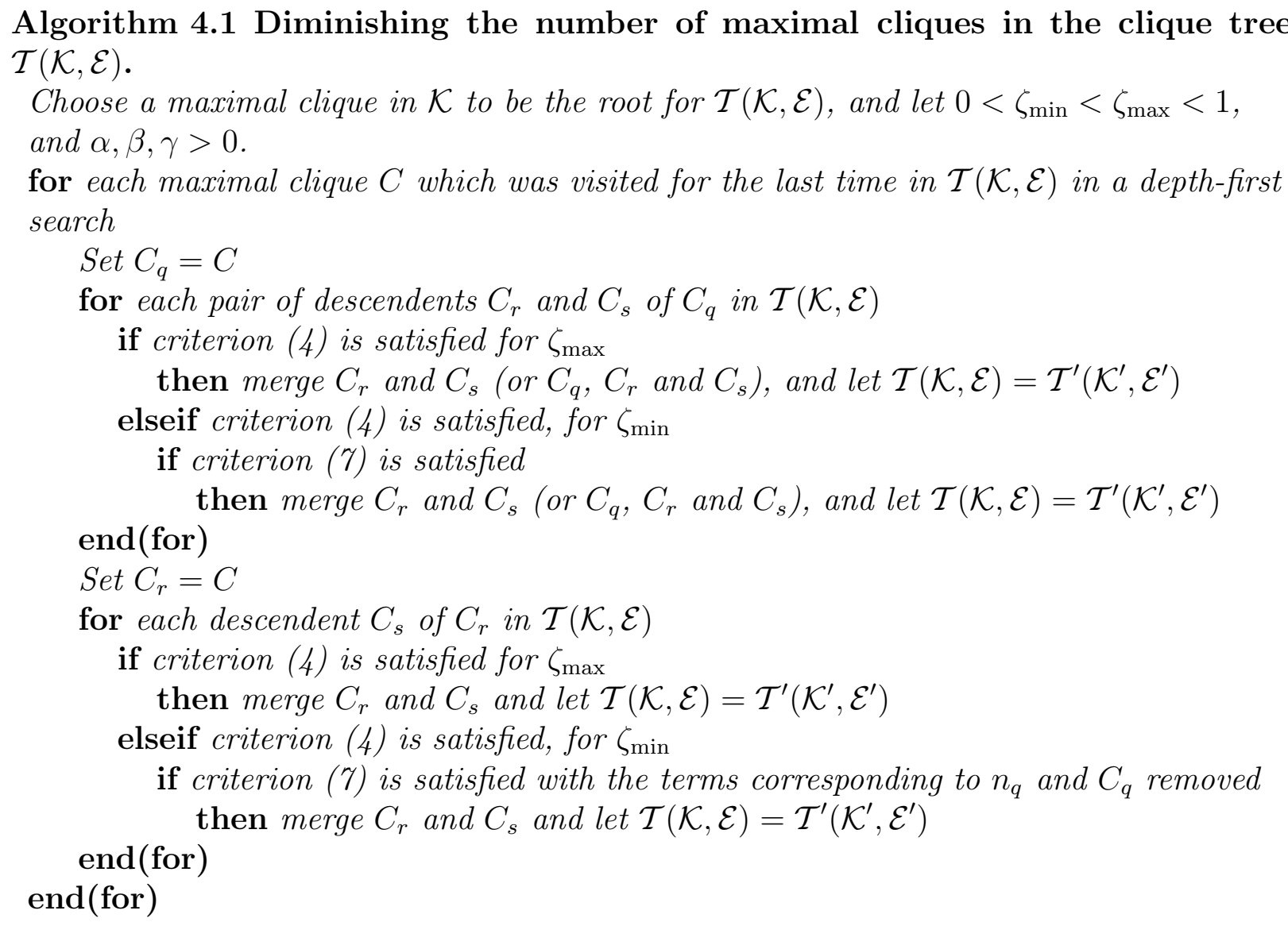

Algorithm 4.1 utilizes the new criterion (7) if $\zeta_{\min } \leq h\left(C_{r}, C_{s}\right)<\zeta_{\max }$. If $h\left(C_{r}, C_{s}\right) \geq$ $\zeta_{\max }$, we automatically decide to merge. If $h\left(C_{r}, C_{s}\right)<\zeta_{\min }$, we do not merge. This strategy avoids excessive evaluation of (7).

As in Algorithm 3.2, it remains a critical question of how we choose the parameters; $\alpha, \beta, \gamma>0$ in Algorithm 4.1, and $\zeta, \eta \in(0,1)$ in Algorithm 3.2.

Although we are mainly focusing on SDPA 6.00, we suppose that the same algorithm and criterion can be adopted for SDPT3 3.02 [23] with the HRVW/KSH/M direction since it also utilizes the formula $\mathcal{F}_{*}[7]$ to compute the SCM, and it has the same complexity order at each iteration of the primal-dual interior-point method. Therefore, we have also included it in our numerical experiments. 


\subsection{Estimating Parameters for the Best Performance}

Estimating parameters $\zeta, \eta \in(0,1)$ in Algorithm 3.2, and $\alpha, \beta, \gamma>0$ in Algorithm 4.1 is not an easy task. In fact, our experience tell us that each SDP has its "best parameter". Nevertheless, we propose the following way to determine the possible best and universal parameters $\zeta, \eta, \alpha, \beta$, and $\gamma$.

We consider four classes of SDP from [16] as our benchmark problems, i.e., norm minimization problems, SDP relaxation of quadratic programs with box constraints, SDP relaxation of max-cut problems over lattice graphs, and SDP relaxation of graph partitioning problems (see Subsection 5.1).

Then using a technic which combines the analysis of variance (ANOVA) [20] and the orthogonal arrays [19] described in [21], we try to estimate the universal parameters. The ANOVA is in fact a well-known method to detect the most significant factors (parameters). However, it is possible to determine the best values for the parameters in the process to compute them. Therefore, repeating ANOVA for different set of parameter values, we can obtain the most possible best parameters for our benchmark problems. In addition, the orthogonal arrays allow us to avoid the experimentations of all possible combinations of parameter values, and even though, obtain the desired result. Details of the method are beyond the objective of this paper and therefore omitted here.

We conducted our experiments at two different computers to verify the sensibility of the parameters: computer A (Pentium III 700MHz with a level 1 data cache of $16 \mathrm{~KB}$, level 2 cache of $1 \mathrm{MB}$, and main memory of $2 \mathrm{~GB}$ ) and computer $\mathrm{B}$ (Athlon $1.2 \mathrm{GHz}$ with a level 1 data cache of $64 \mathrm{~KB}$, level 2 cache of $256 \mathrm{~KB}$, and main memory of $2 \mathrm{~GB}$ ). We obtained the following parameters given at Table 1 for SDPA 6.00 [25] and SDPT3 3.02 [23].

Table 1: Parameters for Algorithms 3.2 and 4.1 at the computers A and B when using SDPA 6.00 and SDPT3 3.02.

\begin{tabular}{c|cc|ccc||cc|ccc} 
& \multicolumn{4}{|c||}{ computer A } & \multicolumn{5}{c}{ computer B } \\
\hline \multicolumn{1}{c|}{ code } & $\zeta$ & $\eta$ & $\alpha$ & $\beta$ & $\gamma$ & $\zeta$ & $\eta$ & $\alpha$ & $\beta$ & $\gamma$ \\
\hline SDPA 6.00 & 0.065 & 0.963 & 0.50 & 36 & 11 & 0.055 & 0.963 & 0.72 & 16 & 9 \\
SDPT3 3.02 & 0.095 & 1.075 & 0.70 & 20 & 46 & 0.085 & 0.925 & 0.58 & 12 & 50 \\
\hline
\end{tabular}

\section{$5 \quad$ Numerical Experiments}

We report in this section the numerical experiments on the performance of proposed versions of the conversion method, i.e., the original version with a new parameter in its heuristic procedure (Subsection 3.2) and the newly proposed one which estimates the flops of each iteration of SDP solvers (Subsection 4.1).

Among the major codes to solve SDPs, we chose the SDPA 6.00 [25] and the SDPT3 3.02 [23]. Both codes are implementations of the primal-dual predictor-corrector infeasible interior-point method. In addition, they use the HRVW/KSH/M search direction and the 
subroutines described in Subsection 4.1 (also [7]) to compute the SCM in which our newly proposed conversion flop estimation version partially relies.

Two different computers were used in the numerical experiments: computer A (Pentium III $700 \mathrm{MHz}$ with a level 1 data cache of $16 \mathrm{~KB}$, level 2 cache of $1 \mathrm{MB}$, and main memory of $2 \mathrm{~GB}$ ) and computer $\mathrm{B}$ (Athlon $1.2 \mathrm{GHz}$ with a level 1 data cache of $64 \mathrm{~KB}$, level 2 cache of $256 \mathrm{~KB}$, and main memory of $2 \mathrm{~GB}$ ). Observe that they have different $\mathrm{CPU}$ chips and foremost, different cache sizes which have a relative effect on the performance of numerical linear algebra subroutines used in each of the codes.

Three different set of SDPs were tested, and they are reported in the next subsections. Subsection 5.1 reports results on the SDPs we used to estimate the parameters (see Subsection 4.2), and the same parameters were used for the SDPs in Subsections 5.2, and 5.3. The other parameters $\kappa$ in (5) was fixed to 2.2, $\zeta_{\min }$ and $\zeta_{\max }$ of Algorithm 4.1 were fixed to 0.035 and 0.98 , respectively.

At the tables that follows, the original problem sizes are given by the number of equality constraints $m$, the number of rows of each block matrices $n$ (where "d" after a number denotes a diagonal matrix), and the sparsity of problems which can be partially understood by the percentage of the aggregate and extended sparsity patterns (Section 2).

In each of numerical result tables, "standard" means the time to solve the corresponding problem by the SDPA 6.00 or SDPT3 3.02 only, "conversion" is the time to solve the equivalent SDP after its conversion by the original version with a new parameter proposed in Subsection 3.2, and "conversion-fe" is the time to solve the equivalent SDP after its conversion by the version proposed in Subsection 4.1. The numbers between parenthesis are the time for the "conversion" and "conversion-fe" themselves. Entries with "=" mean that the converted SDPs became exactly the same as before the conversion. $m_{+}$is the number of equality constraints and $n_{\max }$ gives the sizes of three largest block matrices after the respective conversion.

We utilized the default parameters both for SDPA 6.00 and SDPT3 3.02 excepting $\lambda^{*}$ which was occasionally changed for SDPA 6.00, and OPTIONS.gaptol set to $10^{-7}$ and OPTIONS.cachesize was set according to the computer for SDPT3 3.02. When the solvers fail to solve an instance, we report the relative duality gap denoted by "rg",

$$
\begin{array}{cc}
(\text { for SDPA) } & \text { (for SDPT3) } \\
\frac{\left|\boldsymbol{A}_{0} \bullet \boldsymbol{X}-\sum_{p=1}^{m} b_{p} y_{p}\right|}{\max \left\{1.0,\left(\left|\boldsymbol{A}_{0} \bullet \boldsymbol{X}\right|+\left|\sum_{p=1}^{m} b_{p} y_{p}\right|\right) / 2\right\}}, & \frac{\boldsymbol{X} \bullet \boldsymbol{S}}{\max \left\{1.0,\left(\left|\boldsymbol{A}_{0} \bullet \boldsymbol{X}\right|+\left|\sum_{p=1}^{m} b_{p} y_{p}\right|\right) / 2\right\}},
\end{array}
$$

and/or the feasibility error, denoted by "fea",

(primal feasilibity error for SDPA)

$\max \left\{\left|\boldsymbol{A}_{p} \bullet \boldsymbol{X}-b_{p}\right|: p=1,2, \ldots, m\right\}$,

(primal feasilibity error for SDPT3)

$$
\frac{\sqrt{\sum_{p=1}^{m}\left(\boldsymbol{A}_{p} \bullet \boldsymbol{X}-b_{p}\right)^{2}}}{\max \left\{1.0,\|\boldsymbol{b}\|_{2}\right\}},
$$

(dual feasilibity error for SDPA)

$$
\max \left\{\left|\left[\sum_{p=1}^{m} \boldsymbol{A}_{p} y_{p}+\boldsymbol{S}-\boldsymbol{C}\right]_{i j}\right|: i, j=1,2, \ldots, n\right\},
$$

(dual feasilibity error for SDPT3)

$$
\frac{\left\|\sum_{p=1}^{m} \boldsymbol{A}_{p} y_{p}+\boldsymbol{S}-\boldsymbol{C}\right\|_{2}}{\max \left\{1.0,\|\boldsymbol{C}\|_{2}\right\}},
$$

respectively. For instance "rg" $=2$ means that the relative duality gap is less than $10^{-2}$ and "fea" =p6 means that the primal feasibility error is less than $10^{-6}$. When "rg" and "fea" are less than the required accuracy $10^{-7}$, they are omitted at the tables. 


\subsection{Benchmark Problems}

The sizes of our benchmark SDPs, i.e., norm minimization problems, SDP relaxation of quadratic programs with box constraints, SDP relaxation of max-cut problems over lattice graphs, and SDP relaxation of graph partitioning problems, are shown at Table 2. The original formulation of graph partitioning problems gives a dense aggregate sparsity pattern not allowing us to use the conversion method, and therefore we previously applied an appropriate congruent transformation $[8$, Section 6$]$ to them.

Table 2: Sizes of norm minimization problems, SDP relaxation of quadratic programs with box constraints, SDP relaxation of the maximum cut problems, and SDP relaxation of graph partition problems.

\begin{tabular}{|c|c|c|c|c|}
\hline problem & $m$ & $n$ & aggregate (\%) & extended (\%) \\
\hline norm1 & 11 & 1000 & 0.30 & 0.30 \\
\hline norm2 & 11 & 1000 & 0.50 & 0.50 \\
\hline norm5 & 11 & 1000 & 1.10 & 1.10 \\
\hline norm10 & 11 & 1000 & 2.08 & 2.09 \\
\hline norm 20 & 11 & 1000 & 4.02 & 4.06 \\
\hline norm50 & 11 & 1000 & 9.60 & 9.84 \\
\hline qp3.0 & 1001 & $1001,1000 \mathrm{~d}$ & 0.50 & 2.83 \\
\hline qp3.5 & 1001 & $1001,1000 d$ & 0.55 & 4.56 \\
\hline qp4.0 & 1001 & $1001,1000 d$ & 0.60 & 6.43 \\
\hline $\mathrm{qp} 4.5$ & 1001 & $1001,1000 d$ & 0.66 & 8.55 \\
\hline qp5.0 & 1001 & $1001,1000 \mathrm{~d}$ & 0.70 & 10.41 \\
\hline mcp $2 \times 500$ & 1000 & 1000 & 0.40 & 0.50 \\
\hline mcp $4 \times 250$ & 1000 & 1000 & 0.45 & 0.86 \\
\hline mcp $5 \times 200$ & 1000 & 1000 & 0.46 & 1.03 \\
\hline mсp $8 \times 125$ & 1000 & 1000 & 0.47 & 1.38 \\
\hline mcp $10 \times 100$ & 1000 & 1000 & 0.48 & 1.57 \\
\hline mcp $20 \times 50$ & 1000 & 1000 & 0.49 & 2.12 \\
\hline mcp $25 \times 40$ & 1000 & 1000 & 0.49 & 2.25 \\
\hline gpp $2 \times 500$ & 1001 & 1000 & 0.70 & 0.70 \\
\hline gpp $4 \times 250$ & 1001 & 1000 & 1.05 & 1.10 \\
\hline gpp $5 \times 200$ & 1001 & 1000 & 1.06 & 1.30 \\
\hline gpp $8 \times 125$ & 1001 & 1000 & 1.07 & 2.39 \\
\hline gpp $10 \times 100$ & 1001 & 1000 & 1.07 & 2.94 \\
\hline gpp20×50 & 1001 & 1000 & 1.08 & 4.97 \\
\hline gpp $25 \times 40$ & 1001 & 1000 & 1.08 & 5.31 \\
\hline
\end{tabular}

The discussion henceforth considers the advantages in terms of the computational time.

Tables 3 and 4 give the results for SDPA 6.00 at computer A and B, respectively. For the norm minimization problems, it is advantageous to apply the "conversion". For the SDP relaxations of maximum cut problems and graph partitioning problems, it seems that "conversion-fe" is mostly better than "standard" or "conversion". However, for the SDP 
Table 3: Numerical results on norm minimization problems, SDP relaxation of quadratic programs with box constraints, SDP relaxation of the maximum cut problems, and SDP relaxation of graph partition problems for SDPA 6.00 at computer A.

\begin{tabular}{|c|c|c|c|c|c|c|c|}
\hline \multirow[b]{2}{*}{ problem } & \multirow{2}{*}{$\begin{array}{c}\text { standard } \\
\text { time }(\mathrm{s})\end{array}$} & \multicolumn{3}{|c|}{ conversion } & \multicolumn{3}{|c|}{ conversion-fe } \\
\hline & & $m_{+}$ & $n_{\max }$ & time $(\mathrm{s})$ & $m_{+}$ & $n_{\max }$ & time $(\mathrm{s})$ \\
\hline norm1 & 691.1 & 77 & $16,16,16$ & $2.3(4.3)$ & 58 & $29,29,29$ & $2.9(1.8)$ \\
\hline norm2 & 820.2 & 113 & $31,31,31$ & $4.7(4.4)$ & 71 & $58,58,58$ & $7.4(2.7)$ \\
\hline norm5 & 1047.4 & 206 & $77,77,77$ & $15.4(4.5)$ & 116 & $143,143,143$ & $33.6(9.0)$ \\
\hline norm10 & 1268.8 & 341 & $154,154,154$ & $50.4(5.8)$ & 231 & $286,286,286$ & $138.1(34.2)$ \\
\hline norm 20 & 1631.7 & 641 & $308,308,308$ & $192.6(8.9)$ & 221 & 572,448 & $514.3(182.9)$ \\
\hline norm50 & 2195.5 & 1286 & 770,280 & $1093.0(20.2)$ & 11 & 1000 & $=(20.0)$ \\
\hline qp3.0 & 895.4 & 1373 & $816,22,19$ & $916.1(34.3)$ & 1219 & $864,21,18$ & $847.4(41.8)$ \\
\hline qp 3.5 & 891.5 & 1444 & $844,20,18$ & $1041.5(39.3)$ & 1249 & $875,18,12$ & $890.2(45.2)$ \\
\hline qp4.0 & 891.0 & 1636 & $856,26,20$ & $1294.5(48.2)$ & 1420 & $883,20,13$ & $1084.5(53.4)$ \\
\hline qp4.5 & 891.4 & 1431 & $905,15,10$ & $1163.6(63.2)$ & 1284 & $930,10,9$ & $1028.3(68.4)$ \\
\hline qp5.0 & 892.7 & 1515 & $909,12,11$ & $1206.3(74.4)$ & 1381 & $922,12,11$ & $1045.9(79.4)$ \\
\hline mcp $2 \times 500$ & 822.6 & 1102 & $31,31,31$ & $91.8(1.1)$ & 1051 & $58,58,58$ & $53.0(4.4)$ \\
\hline mср $4 \times 250$ & 719.0 & 1236 & $64,63,63$ & $96.4(2.1)$ & 1204 & $118,116,115$ & $97.5(5.7)$ \\
\hline mcp5 $\times 200$ & 764.9 & 1395 & $91,82,82$ & $153.5(2.5)$ & 1317 & $156,149,146$ & $125.5(6.3)$ \\
\hline mсp $8 \times 125$ & 662.8 & 1343 & $236,155,134$ & 100.7( & 1202 & $240,236,233$ & $72.3(12.3)$ \\
\hline mcp1 & 1.1 & 1547 & $204,172,161$ & $129.0(7.8)$ & 1196 & $301,296,227$ & $88.7(14.7)$ \\
\hline mср20×50 & 653.1 & 1657 & $367,312,307$ & $149.8(19.3)$ & 1552 & $570,367,75$ & $221.5(25.2)$ \\
\hline mср $25 \times 40$ & 690.7 & 1361 & $622,403,5$ & $246.1(30.3)$ & 1325 & 584,441 & $225.2(46.1)$ \\
\hline gpp $2 \times 500$ & 806.1 & 1133 & 7,47 & $77.7(1.7)$ & 1073 & $86,86,86$ & $53.4(5.7)$ \\
\hline gpp $4 \times 250$ & 814.3 & 1181 & 136,7 & $64.4(2.8)$ & 1106 & $143,143,143$ & $56.5(8.1)$ \\
\hline gpp $5 \times 200$ & 807.5 & 1211 & $130,93,93$ & $67.7(3.5)$ & 1106 & $172,172,172$ & $63.8(10.4)$ \\
\hline gpp $8 \times 125$ & 806.1 & 1472 & $170,166,159$ & $119.5(6.8)$ & 1392 & $319,290,272$ & $144.6(14.1)$ \\
\hline gpp10×100 & 798.9 & 1809 & $236,208,203$ & $195.1(10.6)$ & 1263 & $396,339,296$ & $151.2(23.4)$ \\
\hline gpp20×50 & 799.6 & 1679 & $573,443,35$ & $314.5(18.2)$ & 1379 & 566,461 & $293.7(21.1)$ \\
\hline gpp $25 \times 40$ & 808.3 & 1352 & 526,500 & $268.3(39.2)$ & 1904 & 684,353 & $436.3(82.2)$ \\
\hline
\end{tabular}

relaxation of quadratic programs, any conversion is not ideal. This result is particularly intriguing since the superiority of the "conversion" was clear when using SDPA 5.0 [16], and SDPA 6.00 mainly differs from SDPA 5.0 in the numerical linear algebra library where Meschach was replaced by ATLAS/LAPACK in the latest version.

Comparing the results at computers $\mathrm{A}$ and $\mathrm{B}$, they have similar tendencies excepting that it is faster to solve the norm minimization problems at computer $\mathrm{A}$ than computer $\mathrm{B}$ due to its cache size.

Similar results can be seen at Tables 5 and 6 for SDPT3 3.02 at computers A and B. However, in this case, "conversion" or "conversion-fe" is better than "standard" on the SDP relaxation of quadratic programs. Also, the computational time for the norm minimization problems at computer $\mathrm{A}$ is not faster than computer B as it was observed for SDPA 6.00.

We observe that for "conversion-fe", all of converted problems at Tables 3, 4, 5, and 6 become the same, respectively, excepting for "norm1", "norm2", "norm10", and "mcp25×40".

Summing up, preprocessing by "conversion" or "conversion-fe" produces in the best case a speed up of 8 147 times for "norm1" when compared with "standard" even considering the time for the conversion itself. And in the worse case "qp4.0", they take only 2.4 times more than "standard". 
Table 4: Numerical results on norm minimization problems, SDP relaxation of quadratic programs with box constraints, SDP relaxation of the maximum cut problems, and SDP relaxation of graph partition problems for SDPA 6.00 at computer B.

\begin{tabular}{|c|c|c|c|c|c|c|c|}
\hline \multirow[b]{2}{*}{ problem } & \multirow{2}{*}{$\begin{array}{l}\text { standard } \\
\text { time }(\mathrm{s})\end{array}$} & \multicolumn{3}{|c|}{ conversion } & \multicolumn{3}{|c|}{ conversion-fe } \\
\hline & & $m_{+}$ & $n_{\max }$ & time $(\mathrm{s})$ & $m_{+}$ & $n_{\max }$ & time $(\mathrm{s})$ \\
\hline norm1 & 303.9 & 66 & $19,19,19$ & $1.2(1.9)$ & 51 & $29,29,29$ & $1.4(0.7)$ \\
\hline norm2 & 462.0 & 95 & $37,37,37$ & $2.5(1.8)$ & 68 & $58,58,58$ & $3.6(1.3)$ \\
\hline norm5 & 1181.3 & 176 & $91,91,91$ & $9.3(2.3)$ & 116 & $143,143,143$ & $17.4(4.2)$ \\
\hline norm10 & 2410.4 & 286 & $182,182,182$ & $43.2(3.4)$ & 176 & $286,286,286$ & $77.1(14.9)$ \\
\hline norm20 & 2664.5 & 431 & $364,364,312$ & $251.9(6.0)$ & 221 & 572,448 & $1350.2(110.3)$ \\
\hline norm50 & 2970.3 & 1286 & 910,140 & $2632.2(15.4)$ & 11 & 1000 & $=(15.2)$ \\
\hline qp3.0 & 349.4 & 1287 & $843,22,19$ & $493.3(18.5)$ & 1219 & $864,21,18$ & $447.9(20.7)$ \\
\hline qp 3.5 & 348.1 & 1391 & $853,20,18$ & $580.7(22.4)$ & 1249 & $875,18,12$ & $474.6(24.7)$ \\
\hline $\mathrm{qp} 4.0$ & 347.8 & 1601 & $861,26,20$ & $789.2(32.4)$ & 1420 & $883,20,13$ & $631.9(34.6)$ \\
\hline $\mathrm{qp} 4.5$ & 349.0 & 1399 & $915,15,10$ & $626.9(46.9)$ & 1284 & $930,10,9$ & $540.3(49.1)$ \\
\hline qp 5.0 & 347.5 & 1514 & $910,12,11$ & $709.5(59.0)$ & 1381 & $922,12,11$ & $574.6(60.3)$ \\
\hline mcp2 $2 \times 500$ & 268.3 & 1084 & $37,37,37$ & $49.2(0.7)$ & 1051 & $58,58,58$ & $35.1(2.2)$ \\
\hline mсp $4 \times 250$ & 234.2 & 1204 & $85,75,75$ & $56.0(1.2)$ & 1204 & $118,116,115$ & $67.2(2.9)$ \\
\hline mсp5 $\times 200$ & 250.7 & 1295 & $106,96,94$ & $73.0(1.6)$ & 1317 & $156,149,146$ & $95.2(3.2)$ \\
\hline $\operatorname{mcp} 8 \times 125$ & 221.5 & 1340 & $160,155,154$ & $52.7(3.2)$ & 1202 & $240,236,233$ & $36.2(6.8)$ \\
\hline mсp $10 \times 100$ & 233.1 & 1615 & $233,201,187$ & $101.5(4.0)$ & 1196 & $301,296,227$ & $42.1(8.2)$ \\
\hline mср20×50 & 215.6 & 1330 & $581,392,62$ & $84.2(8.3)$ & 1552 & $570,367,75$ & $102.8(14.6)$ \\
\hline $\operatorname{mcp} 25 \times 40$ & 228.4 & 1325 & 567,458 & $87.2(18.3)$ & 1406 & 650,378 & $100.5(33.5)$ \\
\hline gpp $2 \times 500$ & 265.5 & 1109 & $55,55,55$ & $44.7(1.0)$ & 1073 & $86,86,86$ & $31.9(2.8)$ \\
\hline gpp $4 \times 250$ & 267.1 & 1151 & $140,91,91$ & $34.7(1.8)$ & 1106 & $143,143,143$ & $29.9(4.4)$ \\
\hline gpp5×200 & 265.3 & 1169 & $168,110,110$ & $34.0(2.3)$ & 1106 & $172,172,172$ & $32.5(5.5)$ \\
\hline gpp $8 \times 125$ & 265.0 & 1337 & $202,177,176$ & $49.2(4.7)$ & 1392 & $319,290,272$ & $93.3(7.6)$ \\
\hline gpp10×100 & 253.4 & 1536 & $277,277,242$ & $107.9(7.0)$ & 1263 & $396,339,296$ & $62.0(13.5)$ \\
\hline gpp $20 \times 50$ & 262.4 & 2030 & $512,491,39$ & $179.7(9.4)$ & 1379 & 566,461 & $112.3(10.9)$ \\
\hline gpp $25 \times 40$ & 264.1 & 1352 & 526,500 & $103.2(22.1)$ & 1904 & 689,353 & $183.7(47.6)$ \\
\hline
\end{tabular}

\subsection{SDPLIB Problems}

The next set of problems are from the SDPLIB 1.2 collection [4]. We selected the problems which have sparse aggregate patterns including the ones after the congruent transformation [8, Section 6] like "equalG", "gpp", and "theta" problems. We excluded the largest instances. Problem sizes and sparsity information are shown at Table 7. Observe that in several cases, the fill-in effect causes the extended sparsity patterns to become much denser than the corresponding aggregate sparsity patterns.

We can observe from the numerical results at Tables 8, 9, 10, and 11 that the conversion method is advantageous when the extended sparsity patterns are less than 5\%, which is the case for "equalG11", "maxG11", "maxG32", "mcp124-1", "mcp250-1", "mcp500-1", "qpG11", "qpG51", and "thetaG11". The exception are "mcp124-1" and "mcp250-1" at Table 10, and "mcp124-1" at Table 11. In particular, it is difficult to say which version of the conversion method is ideal in general, but it seems that "conversion" is particularly better for "maxG32", and "conversion-fe" is better for "equalG11", "maxG11", and "qpG11".

Once again, the converted problems under the columns "conversion-fe" are exactly the same for the Tables 8, 9, 10, and 11 respectively, excepting "arch" problems, "equalG11", "gpp124-1", "gpp124-2", "maxG11", "mcp250-4", "mcp500-1", "qpG11", and "ss30".

For "qpG11" we have a speed up of 6.4 19.3 times when preprocessed by "conversion" or "conversion-fe" even considering the time for the conversion itself. On the other hand, 
Table 5: Numerical results on norm minimization problems, SDP relaxation of quadratic programs with box constraints, SDP relaxation of the maximum cut problems, and SDP relaxation of graph partition problems for SDPT3 3.02 at computer A.

\begin{tabular}{|c|c|c|c|c|c|c|c|}
\hline \multirow[b]{2}{*}{ problem } & standard & \multicolumn{3}{|c|}{ conversion } & \multicolumn{3}{|c|}{ conversion-fe } \\
\hline & time $(\mathrm{s}) \mathrm{rg}$ fea & $m_{+}$ & $n_{\max }$ & time $(\mathrm{s})$ & $m_{+}$ & $n_{\max }$ & time $(\mathrm{s})$ \\
\hline norm1 & 234.7 & 110 & $11,11,11$ & $10.5(6.0)$ & 53 & $29,29,29$ & $20.9(1.5) \mathrm{p} 6$ \\
\hline norm2 & 330.7 & 158 & $22,22,22$ & $23.9(5.1) \mathrm{p} 6$ & 68 & $58,58,58$ & $26.2(2.6)$ \\
\hline norm5 & 418.5 & 311 & $53,53,53$ & $51.5(4.9)$ p 6 & 116 & $143,143,143$ & $59.3(8.8) \mathrm{p} 6$ \\
\hline norm10 & 507.7 & 561 & $106,106,106$ & 85.4 (6.1) p6 & 176 & $286,286,286$ & $108.3(33.7)$ \\
\hline norm20 & 686.2 & 1061 & $211,211,211$ & $178.4(9.3)$ & 221 & 572,448 & $325.2(181.1)$ \\
\hline norm50 & 1295.5 & 1286 & 527,523 & $730.2(20.9)$ & 11 & 1000 & $=(20.3)$ \\
\hline qp3.0 & 466.3 & 1717 & $769,57,22$ & $333.6(31.0)$ & 1219 & $864,21,18$ & $336.8(41.8)$ \\
\hline qp3.5 & 470.3 & 1616 & $810,20,18$ & $324.8(36.7)$ & 1249 & $875,18,12$ & $328.6(44.9)$ \\
\hline qp4.0 & 500.6 & 1913 & $835,26,22$ & $436.0(46.6)$ & 1420 & $883,13,10$ & $397.7(53.5)$ \\
\hline qp4.5 & 492.9 & 1681 & $875,15,14$ & $449.0(61.3)$ & 1284 & $930,10,9$ & $469.0(68.4)$ \\
\hline qp5.0 & 487.6 & 1929 & $878,20,20$ & $460.6(71.7)$ & 1381 & $922,12,11$ & $419.4(79.4)$ \\
\hline $\mathrm{mcp} 2 \times 500$ & $264.0 \quad 2 \quad \mathrm{p} 2$ & 1144 & $22,22,22$ & $24.9(0.9)$ & 1051 & $58,58,58$ & $66.7(4.5)$ \\
\hline $\operatorname{mcp} 4 \times 250$ & 297.7 & 1334 & $72,45,45$ & $108.5(1.6)$ & 1204 & $118,116,115$ & $67.6(5.7)$ \\
\hline $\operatorname{mcp} 5 \times 200$ & 307.5 & 1315 & $92,68,58$ & $91.5(2.0)$ & 1317 & $156,149,146$ & $79.1(6.2)$ \\
\hline $\operatorname{mcp} 8 \times 125$ & 272.4 & 1494 & $95,94,94$ & $89.6(3.5)$ & 1202 & $240,236,233$ & $66.5(12.3)$ \\
\hline mcp $10 \times 100$ & 295.4 & 1616 & $150,126,114$ & $92.4(4.2)$ & 1196 & $301,296,227$ & $66.7(14.7)$ \\
\hline $\operatorname{mcp} 20 \times 50$ & 270.4 & 1630 & $349,252,247$ & $116.8(10.9)$ & 1552 & $570,367,75$ & $143.7(25.2)$ \\
\hline $\operatorname{mcp} 25 \times 40$ & 270.2 & 2031 & $328,307,280$ & $179.0(18.0)$ & 1406 & 650,378 & $151.0(58.9)$ \\
\hline gpp2×500 & 432.5 & 1205 & $32,32,32$ & $136.1(1.3)$ & 1073 & $86,86,86$ & $75.9(5.6)$ \\
\hline gpp $4 \times 250$ & 436.8 & 1301 & $53,53,53$ & $105.4(2.1)$ & 1106 & $143,143,143$ & $78.1(8.1)$ \\
\hline gpp $5 \times 200$ & 414.8 & 1337 & $72,64,64$ & $98.4(2.6)$ & 1106 & $172,172,172$ & $87.7(10.4)$ \\
\hline gpp $8 \times 125$ & 430.0 & 1790 & $183,126,121$ & $137.4(5.2)$ & 1392 & $319,290,272$ & $127.2(14.1)$ \\
\hline gpp $10 \times 100$ & 402.9 & 1824 & $188,158,150$ & $139.3(7.0)$ & 1263 & $396,339,296$ & $129.6(23.3)$ \\
\hline gpp $20 \times 50$ & 383.4 & 2599 & $338,298,287$ & $419.7(15.7)$ & 1379 & 566,461 & $184.7(21.2)$ \\
\hline gpp $25 \times 40$ & 388.0 & 1703 & $411,380,261$ & $167.8(21.4)$ & 1904 & 689,353 & $307.8(82.2)$ \\
\hline
\end{tabular}

the worse case goes to "mcp250-1" which takes only 1.6 times more than "standard" when restricting problems with less than $5 \%$ on their extended sparsity patterns.

\subsection{Structural Optimization Problems}

The last set of problems are from the structural optimization [12] which have sparse aggregate sparsity patterns. Problem sizes and sparsity information are shown at Table 12.

The numerical results for these four classes of problems for SDPA 6.00 and SDPT3 3.02 at computers $\mathrm{A}$ and $\mathrm{B}$ are shown at Tables 13, 14, 15, and 16. Entries with "M" means out of memory and "*" means fail to solve.

Among these four classes, the conversion method is only advantageous for the "shmup" problems. In particular, "conversion" and "conversion-fe" have similar performances over "standard" for SDPA 6.00 at computers A and B (Tables 13 and 14), while "conversion" is better than "conversion-fe" and "standard" (excepting "shmup5") for SDPT3 3.02 at computers A and B (Tables 15 and 16).

We observe here that both SDPA 6.00 and SDPT3 3.02 have some difficult to solve the converted problems, i.e., "conversion" and "conversion-fe", and get the same accuracy as "standard", suggesting that the conversion itself can sometimes cause some negative effect when solving by interior-point methods. In some cases like "vibra5" for "conversionfe", SDPT3 3.02 fails to solve them (Tables 15 and 16). However, these structural optimal 
Table 6: Numerical results on norm minimization problems, SDP relaxation of quadratic programs with box constraints, SDP relaxation of the maximum cut problems, and SDP relaxation of graph partition problems for SDPT3 3.02 at computer B.

\begin{tabular}{|c|c|c|c|c|c|c|c|c|}
\hline \multirow[b]{2}{*}{ problem } & standard & \multicolumn{3}{|c|}{ conversion } & \multicolumn{4}{|c|}{ conversion-fe } \\
\hline & time $(\mathrm{s}) \mathrm{rg}$ fea & $m_{+}$ & $n_{\max }$ & time $(\mathrm{s}) \quad$ fea & $m_{+}$ & $n_{\max }$ & time $(\mathrm{s})$ & rg fea \\
\hline norm1 & 115.2 & 101 & $12,12,12$ & $7.2(2.5) \mathrm{p} 5$ & 53 & $29,29,29$ & $14.3(0.7)$ & p6 \\
\hline norm2 & 162.1 & 146 & $24,24,24$ & $18.1(2.1)$ & 68 & $58,58,58$ & $15.8(1.3)$ & 6 \\
\hline norm5 & 209.0 & 281 & $59,59,59$ & $29.1(2.5) \mathrm{p} 6$ & 116 & $143,143,143$ & $36.9(4.2)$ & p6 \\
\hline norm10 & 259.4 & 506 & $118,118,118$ & $51.0(3.5)$ p6 & 176 & $286,286,286$ & $69.5(15.0)$ & \\
\hline norm20 & 414.3 & 851 & $236,236,236$ & $112.1(6.1)$ & 221 & 572,448 & $180.9(110.5)$ & \\
\hline norm50 & 746.6 & 1286 & 589,461 & $446.0(15.6)$ & 11 & 1000 & $=(15.4)$ & \\
\hline qp3.0 & 260.1 & 1397 & $807,22,19$ & $177.9(17.1)$ & 1219 & $864,21,18$ & $191.4(20.8)$ & \\
\hline qp3.5 & 258.9 & 1591 & $806,20,18$ & $180.4(20.7)$ & 1249 & $875,18,12$ & $188.4(24.8)$ & \\
\hline qp 4.0 & 275.0 & 1947 & $823,26,22$ & $242.5(31.0)$ & 1420 & $883,20,13$ & $227.3(34.4)$ & \\
\hline qp4.5 & 267.8 & 1613 & $886,15,14$ & $251.5(45.4)$ & 1284 & $930,10,9$ & $260.0(48.5)$ & \\
\hline qp5.0 & 265.6 & 1856 & $887,20,20$ & $256.5(57.9)$ & 1381 & $922,12,11$ & $233.3(60.9)$ & \\
\hline $\mathrm{mcp} 2 \times 500$ & 140.42 p2 & 1132 & $32,24,24$ & $14.5(0.5)$ & 1051 & $58,58,58$ & $36.4(2.2)$ & \\
\hline $\operatorname{mcp} 4 \times 250$ & 160.8 & 1398 & $51,51,49$ & $59.6(0.9)$ & 1204 & $118,116,115$ & $37.3(2.9)$ & \\
\hline $\operatorname{mcp} 5 \times 200$ & 165.2 & 1476 & $67,66,65$ & $52.6(1.1)$ & 1317 & $156,149,146$ & $44.8(3.2)$ & \\
\hline $\operatorname{mcp} 8 \times 125$ & 148.7 & 1421 & $138,114,111$ & $44.3(2.3)$ & 1202 & $240,236,233$ & $40.1(6.8)$ & \\
\hline mcp $10 \times 100$ & 160.9 & 1764 & $171,167,141$ & $53.8(2.8)$ & 1196 & $301,296,227$ & $42.4(8.2)$ & \\
\hline mcp $20 \times 50$ & 147.6 & 1801 & $297,280,252$ & $73.7(5.9)$ & 1552 & $570,367,75$ & $81.7(14.6)$ & \\
\hline $\operatorname{mcp} 25 \times 40$ & 147.9 & 2112 & $346,328,307$ & $97.6(11.7)$ & 1406 & 650,378 & $86.8(33.4)$ & \\
\hline gpp $2 \times 500$ & 233.8 & 1181 & $36,36,36$ & $67.8(0.8)$ & 1073 & $86,86,86$ & $42.3(2.9)$ & \\
\hline gpp $4 \times 250$ & 235.8 & 1271 & $59,59,59$ & $51.3(1.3)$ & 1106 & $143,143,143$ & $43.7(4.4)$ & \\
\hline gpp $5 \times 200$ & 225.9 & 1295 & $90,71,71$ & $49.7(1.6)$ & 1106 & $172,172,172$ & $51.0(5.5)$ & \\
\hline gpp $8 \times 125$ & 232.7 & 1645 & $132,131,130$ & $70.5(3.2)$ & 1392 & $319,290,272$ & $84.1(7.6)$ & \\
\hline gpp10×100 & 218.1 & 1949 & $186,183,165$ & $98.8(4.8)$ & 1263 & $396,339,296$ & $84.5(13.5)$ & \\
\hline gpp $20 \times 50$ & 209.9 & 2347 & $370,352,319$ & $166.3(15.0)$ & 1379 & 566,461 & $106.6(10.9)$ & \\
\hline gpp $25 \times 40$ & 216.0 & 1703 & $411,380,261$ & $96.2(12.5)$ & 1904 & 689,353 & $176.2(47.4)$ & \\
\hline
\end{tabular}

problems are difficult to solve by their nature (see "rg" and "fea" columns under "standard").

Once again, the converted problems under the columns "conversion-fe" have similar sizes. In particular the converted problems are exactly the same for Tables 13, 14, 15, and 16 for "buck5", "shmup3 5", "trto4 5" and "vibra5", respectively.

Although it is difficult to make direct comparisons due to the difference in accuracies, it seems in general that "conversion-fe" is better than "conversion" for worse case scenarios when preprocessing fails.

\section{Concluding and Further Remarks}

As we stated in Introduction, the conversion method is a preprocessing phase of SDP solvers for sparse and large-scale SDPs. We slightly improved the original version [16] here, and proposed a new version of the conversion method which attempts to produce the best equivalent SDP in terms of shortening the computational time. A flop estimation function was introduced to be used in the heuristic routine in the new version. Extensive numerical computation using SDPA 6.00 and SDPT3 3.02 were conducted comparing the computational time for different sets of SDPs on different computers using the original version of the conversion, "conversion", the flop estimation version, "conversion-fe", and SDPs without preprocessing, "standard". In some cases, the results were astonishing: "norm1" became 
Table 7: Sizes of SDPLIB problems.

\begin{tabular}{|c|c|c|c|c|}
\hline problem & $m$ & $n$ & aggregate (\%) & extended (\%) \\
\hline $\operatorname{arch} 0$ & 174 & $161,174 \mathrm{~d}$ & 11.44 & 24.18 \\
\hline $\operatorname{arch} 2$ & 174 & $161,174 \mathrm{~d}$ & 12.93 & 23.34 \\
\hline $\operatorname{arch} 4$ & 174 & $161,174 \mathrm{~d}$ & 12.93 & 23.34 \\
\hline $\operatorname{arch} 8$ & 174 & $161,174 \mathrm{~d}$ & 12.93 & 23.34 \\
\hline equalG11 & 801 & 801 & 1.24 & (A) 4.32, (B) 4.40 \\
\hline equalG51 & 1001 & 1001 & 4.59 & (A) 52.99, (B) 53.37 \\
\hline gpp100 & 101 & 100 & 20.34 & 61.66 \\
\hline gpp124-1 & 125 & 124 & 9.53 & 33.18 \\
\hline gpp124-2 & 125 & 124 & 16.70 & 56.26 \\
\hline gpp124-3 & 125 & 124 & 28.12 & 74.26 \\
\hline gpp124-4 & 125 & 124 & 44.61 & 89.19 \\
\hline gpp250-1 & 251 & 250 & 5.27 & 31.02 \\
\hline gрp250-2 & 251 & 250 & 8.51 & 52.00 \\
\hline gрp250-3 & 251 & 250 & 16.09 & 72.31 \\
\hline gpp250-4 & 251 & 250 & 26.84 & 84.98 \\
\hline gpp500-1 & 501 & 500 & 2.56 & 28.45 \\
\hline gpp500-2 & 501 & 500 & 4.42 & 46.54 \\
\hline gpp500-3 & 501 & 500 & 7.72 & 66.25 \\
\hline gpp500-4 & 501 & 500 & 15.32 & 83.06 \\
\hline $\operatorname{maxG11}$ & 800 & 800 & 0.62 & 2.52 \\
\hline $\operatorname{maxG} 32$ & 2000 & 2000 & 0.25 & 1.62 \\
\hline $\operatorname{maxG51}$ & 1000 & 1000 & 1.28 & 13.39 \\
\hline mcp100 & 100 & 100 & 6.38 & 19.88 \\
\hline mcp124-1 & 124 & 124 & 2.74 & 4.75 \\
\hline mсp124-2 & 124 & 124 & 4.94 & 16.99 \\
\hline mcp124-3 & 124 & 124 & 8.87 & 38.20 \\
\hline mcp124-4 & 124 & 124 & 17.34 & 64.58 \\
\hline mcp250-1 & 250 & 250 & 1.46 & 3.65 \\
\hline mсp250-2 & 250 & 250 & 2.36 & 14.04 \\
\hline mсp250-3 & 250 & 250 & 4.51 & 34.09 \\
\hline mсp250-4 & 250 & 250 & 8.15 & 57.10 \\
\hline mсp500-1 & 500 & 500 & 0.70 & 2.13 \\
\hline mсp500-2 & 500 & 500 & 1.18 & 10.78 \\
\hline mсp500-3 & 500 & 500 & 2.08 & 27.94 \\
\hline mсp500-4 & 500 & 500 & 4.30 & 52.89 \\
\hline qpG11 & 800 & 1600 & 0.19 & 0.68 \\
\hline qpG51 & 1000 & 2000 & 0.35 & 3.36 \\
\hline ss 30 & 132 & $294,132 \mathrm{~d}$ & 8.81 & 18.71 \\
\hline theta1 & 104 & 50 & 32.24 & 57.68 \\
\hline theta2 & 498 & 100 & 36.08 & 77.52 \\
\hline theta3 & 1106 & 150 & 35.27 & 85.40 \\
\hline theta4 & 1949 & 200 & 34.71 & 85.89 \\
\hline theta5 & 3028 & 250 & 34.09 & 89.12 \\
\hline theta 6 & 4375 & 300 & 34.42 & 90.36 \\
\hline thetaG11 & 2401 & 801 & 1.62 & 4.92 \\
\hline thetaG51 & 6910 & 1001 & 4.93 & 53.65 \\
\hline
\end{tabular}

(A): computer A, (B): computer B. 
Table 8: Numerical results on SDPLIB problems for SDPA 6.00 at computer A.

\begin{tabular}{|c|c|c|c|c|c|c|c|c|c|}
\hline \multirow[b]{2}{*}{ problem } & standard & \multicolumn{4}{|c|}{ conversion } & \multicolumn{4}{|c|}{ conversion-fe } \\
\hline & time $(\mathrm{s}) \mathrm{rg}$ fea & $m_{+}$ & $n_{\max }$ & time $(\mathrm{s})$ & rg fea & $m_{+}$ & $n_{\max }$ & time $(\mathrm{s})$ & rg fea \\
\hline $\operatorname{arch} 0$ & 13.7 & 174 & $161,174 \mathrm{~d}$ & $=(0.4)$ & & 552 & $124,64,174 \mathrm{~d}$ & $21.5(0.6)$ & $6 \mathrm{p} 6$ \\
\hline $\operatorname{arch} 2$ & 15.6 & 174 & $161,174 \mathrm{~d}$ & $=(0.4)$ & & 405 & $100,82,174 \mathrm{~d}$ & $12.6(0.5)$ & $5 \mathrm{p} 5$ \\
\hline $\operatorname{arch} 4$ & 15.6 & 174 & $161,174 \mathrm{~d}$ & $=(0.4)$ & & 405 & $100,82,174 \mathrm{~d}$ & $12.4(0.4)$ & 5 \\
\hline $\operatorname{arch} 8$ & 17.46 & 174 & $161,174 \mathrm{~d}$ & $=(0.4)$ & & 405 & $100,82,174 \mathrm{~d}$ & $12.5(0.5)$ & $4 \mathrm{p} 4$ \\
\hline equalG11 & 455.2 & 1064 & $408,408,9$ & $158.1(14.3)$ & $5 \mathrm{p} 6$ & 1314 & $219,218,209$ & $82.9(7.7)$ & 5 \\
\hline equalG51 & 865.1 & 2682 & $998,52,29$ & $1234.6(679.6)$ & 6 & 1407 & 1000,29 & $900.5(681.3)$ & \\
\hline gpp100 & 1.2 & 101 & 100 & $=(0.2)$ & & 101 & 100 & $=(0.2)$ & \\
\hline gpp124-1 & 2.2 & 125 & 124 & $=(0.2)$ & & 161 & 122,10 & $2.2(0.2)$ & 6 \\
\hline gpp124-2 & 2.1 & 146 & 123,7 & $2.3(0.4)$ & & 146 & 123,7 & $2.1(0.4)$ & \\
\hline gpp124-3 & 2.0 & 125 & 124 & $=(0.5)$ & & 125 & 124 & $=(0.5)$ & \\
\hline gpp124-4 & 2.1 & 125 & 124 & $=(0.7)$ & & 125 & 124 & $=(0.7)$ & \\
\hline gpp250-1 & 14.9 & 272 & 249,7 & $16.9(1.6)$ & & 251 & 250 & $=(1.8)$ & \\
\hline gрp250-2 & 14.6 & 251 & 250 & $=(3.3)$ & & 251 & 250 & $=(3.4)$ & \\
\hline gpp250-3 & 13.5 & 251 & 250 & $=(5.0)$ & & 251 & 250 & $=(5.0)$ & \\
\hline gpp250-4 & 13.3 & 251 & 250 & $=(6.9)$ & & 251 & 250 & $=(6.9)$ & \\
\hline gpp500-1 & 125.5 & 830 & $493,17,13$ & $114.0(16.1)$ & 5 & 537 & 499,9 & $124.0(16.7)$ & 5 \\
\hline gpp500-2 & 115.1 & 1207 & $496,30,26$ & $132.4(41.4)$ & 5 & 501 & 500 & $=(37.2)$ & \\
\hline gpp500-3 & 108.4 & 1005 & $498,27,18$ & $127.4(56.7)$ & 5 & 654 & 499,18 & $111.0(57.1)$ & 6 \\
\hline gpp500-4 & 99.0 & 501 & 500 & $=(88.7)$ & & 501 & 500 & $=(88.8)$ & \\
\hline $\operatorname{maxG11}$ & 343.7 & 972 & $408,403,13$ & $113.3(10.7)$ & & 1208 & $216,216,208$ & $60.8(8.2)$ & \\
\hline $\operatorname{maxG} 32$ & 5526.4 & 2840 & $1021,1017,5$ & $1704.3(274.5)$ & & 2000 & 2000 & $=(358.5)$ & \\
\hline $\max G 51$ & 651.8 & 2033 & $957,16,15$ & $867.6(84.4)$ & & 1677 & $971,15,15$ & $756.8(88.5)$ & \\
\hline mcp100 & 0.8 & 123 & $93,5,5$ & $0.9(0.1)$ & & 107 & $95,5,3$ & $0.8(0.1)$ & \\
\hline mcp124-1 & 1.6 & 129 & $108,4,2$ & $1.2(0.1)$ & & 184 & $60,48,13$ & $0.9(0.1)$ & \\
\hline mcp124-2 & 1.5 & 163 & $117,5,5$ & $1.6(0.1)$ & & 144 & $120,5,4$ & $1.5(0.2)$ & \\
\hline mcp124-3 & 1.5 & 160 & $122,7,6$ & $1.6(0.2)$ & & 124 & 124 & $=(0.3)$ & \\
\hline mcp124-4 & 1.5 & 124 & 124 & $=(0.4)$ & & 124 & 124 & $=(0.4)$ & \\
\hline mсp250-1 & 10.9 & 268 & $217,7,3$ & $8.5(0.5)$ & & 401 & $176,58,7$ & $8.3(0.5)$ & \\
\hline mср250-2 & 10.6 & 342 & $233,12,5$ & $11.9(0.9)$ & & 327 & $237,12,5$ & $11.2(1.0)$ & \\
\hline mср250-3 & 10.6 & 444 & $243,11,11$ & $14.1(1.9)$ & & 277 & $247,6,4$ & $10.6(2.1)$ & \\
\hline mср250-4 & 10.5 & 305 & 249,11 & $11.3(3.6)$ & & 305 & 249,11 & $11.6(3.6)$ & \\
\hline mср500-1 & 87.6 & 545 & $403,10,10$ & $63.1(3.6)$ & & 1271 & $324,124,10$ & $115.2(3.3)$ & \\
\hline mcp500-2 & 87.9 & 899 & $436,14,12$ & $127.7(6.4)$ & & 784 & $449,15,9$ & $109.2(7.3)$ & \\
\hline mcp500-3 & 82.4 & 1211 & $477,18,16$ & $155.6(17.5)$ & & 1019 & $482,16,13$ & $128.2(18.2)$ & \\
\hline mcp500-4 & 82.6 & 2013 & $491,20,20$ & $246.8(46.1)$ & & 758 & $498,18,15$ & $89.5(46.8)$ & \\
\hline qpG11 & 2612.5 & 946 & $419,396,5$ & $181.6(15.6)$ & & 1208 & $219,216,213$ & $187.6(12.3)$ & \\
\hline qpG51 & 5977.3 & 2243 & $947,16,15$ & $1830.4(88.4)$ & & 1838 & $965,16,15$ & $1357.1(99.8)$ & \\
\hline ss30 & 99.1 & 132 & $294,132 \mathrm{~d}$ & $=(1.1)$ & & 1035 & $171,165,132 \mathrm{~d}$ & $67.0(1.5)$ & $4 \mathrm{p} 6$ \\
\hline theta1 & 0.4 & 104 & 50 & $=(0.0)$ & & 104 & 50 & $=(0.0)$ & \\
\hline theta2 & 7.8 & 498 & 100 & $=(0.5)$ & & 498 & 100 & $=(0.5)$ & \\
\hline theta3 & 43.8 & 1106 & 150 & $=(2.1)$ & & 1106 & 150 & $=(2.2)$ & \\
\hline theta4 & 184.96 & 1949 & 200 & $=(6.2)$ & & 1949 & 200 & $=(6.7)$ & \\
\hline theta5 & $581.0 \quad 6$ & 3028 & 250 & $=(15.0)$ & & 3028 & 250 & $=(15.7)$ & \\
\hline theta6 & 1552.96 & 4375 & 300 & $=(31.1)$ & & 4375 & 300 & $=(32.2)$ & \\
\hline thetaG11 & 1571.8 & 2743 & $315,280,242$ & $852.8(31.7)$ & & 2572 & 417,402 & $937.9(51.1)$ & \\
\hline thetaG51 & 24784.73 p5 & 7210 & 1000,25 & $*(817.8)$ & & 6910 & 1001 & $=(855.9)$ & \\
\hline
\end{tabular}

8 147 times faster, and "qpG11" became 6 19 faster when compared with "standard".

Unfortunately, it seems that each SDP prefers one of the versions of the conversion method. However, we can say in general that both conversions are advantageous to use when the extended sparsity pattern is less than $5 \%$, and even in abnormal cases, like in structural optimization problems where getting the feasibility is difficult, the computational time takes at most 4 times more than solving without any conversion.

In practical matters, it is really worth to preprocess by "conversion" or "conversion-fe" 
Table 9: Numerical results on SDPLIB problems for SDPA 6.00 at computer B.

\begin{tabular}{|c|c|c|c|c|c|c|c|c|c|c|}
\hline \multirow[b]{2}{*}{ problem } & \multicolumn{2}{|c|}{ standard } & \multicolumn{4}{|c|}{ conversion } & \multicolumn{4}{|c|}{ conversion-fe } \\
\hline & time $(\mathrm{s})$ & rg fea & $m_{+}$ & $n_{\max }$ & time $(\mathrm{s})$ & $\mathrm{rg}$ & $m_{+}$ & $n_{\max }$ & time $(\mathrm{s})$ & $\mathrm{rg}$ \\
\hline $\operatorname{arch0}$ & 6.8 & & 174 & $161,174 \mathrm{~d}$ & $=(0.2)$ & & 174 & $161,174 \mathrm{~d}$ & $=(0.3)$ & \\
\hline $\operatorname{arch} 2$ & 7.7 & & 174 & $161,174 \mathrm{~d}$ & $=(0.2)$ & & 174 & $161,174 d$ & $=(0.3)$ & \\
\hline $\operatorname{arch} 4$ & 7.6 & & 174 & $161,174 d$ & $=(0.2)$ & & 174 & $161,174 d$ & $=(0.3)$ & \\
\hline $\operatorname{arch} 8$ & 8.2 & 6 & 174 & $161,174 d$ & $=(0.2)$ & 6 & 174 & $161,174 \mathrm{~d}$ & $=(0.3)$ & 6 \\
\hline equalG11 & 153.5 & & 1008 & $409,409,9$ & $57.0(8.0)$ & 5 & 972 & 410,409 & $51.7(8.5)$ & 5 \\
\hline equalG51 & 284.3 & & 2682 & $998,52,29$ & $498.8(762.4)$ & 6 & 1407 & 1000,29 & $318.9(763.6)$ & 6 \\
\hline gpp100 & 0.5 & & 101 & 100 & $=(0.2)$ & & 101 & 100 & $=(0.2)$ & \\
\hline gpp124-1 & 0.9 & & 125 & 124 & $=(0.1)$ & & 125 & 124 & $=(0.2)$ & \\
\hline gpp124-2 & 0.9 & & 146 & 123,7 & $1.0(0.3)$ & & 125 & 124 & $=(0.3)$ & \\
\hline gpp124-3 & 0.9 & & 125 & 124 & $=(0.4)$ & & 125 & 124 & $=(0.4)$ & \\
\hline gpp124-4 & 0.9 & & 125 & 124 & $=(0.6)$ & & 125 & 124 & $=(0.6)$ & \\
\hline gpp250-1 & 6.4 & & 272 & 249,7 & $6.5(1.3)$ & & 251 & 250 & $=(1.4)$ & \\
\hline gpp250-2 & 6.1 & & 251 & 250 & $=(3.2)$ & & 251 & 250 & $=(3.2)$ & \\
\hline gpp250-3 & 5.9 & & 251 & 250 & $=(4.9)$ & & 251 & 250 & $=(4.9)$ & \\
\hline gpp250-4 & 5.7 & & 251 & 250 & $=(7.0)$ & & 251 & 250 & $=(7.1)$ & \\
\hline gpp500-1 & 45.6 & & 752 & $494,17,11$ & $54.4(14.9)$ & 5 & 537 & 499,9 & $45.6(15.2)$ & 5 \\
\hline gpp500-2 & 42.0 & & 801 & 498,26 & 47.7 (41.6) & 6 & 501 & 500 & $=(39.2)$ & \\
\hline gpp500-3 & 39.5 & & 1005 & $498,27,18$ & $57.0(61.8)$ & 6 & 654 & 499,18 & $42.0(62.1)$ & 6 \\
\hline gpp500-4 & 36.1 & & 501 & 500 & $=(98.7)$ & & 501 & 500 & $=(99.0)$ & \\
\hline $\max G 11$ & 118.6 & & 936 & 408,408 & $44.0(6.0)$ & & 1072 & $408,216,208$ & $37.6(5.8)$ & \\
\hline $\max G 32$ & 1652.1 & & 2820 & 1022,1018 & $618.9(150.4)$ & & 2000 & 2000 & $=(182.2)$ & \\
\hline $\max G 51$ & 216.2 & & 1868 & $964,16,15$ & $432.6(73.9)$ & & 1677 & $971,15,15$ & $345.9(75.6)$ & \\
\hline mcp100 & 0.4 & & 107 & $95,5,3$ & $0.4(0.0)$ & & 107 & $95,5,3$ & $0.4(0.1)$ & \\
\hline mcp124-1 & 0.7 & & 129 & $108,4,2$ & $0.6(0.0)$ & & 184 & $60,48,13$ & $0.5(0.0)$ & \\
\hline mcp124-2 & 0.7 & & 157 & $118,5,5$ & $0.8(0.1)$ & & 144 & $120,5,4$ & $0.7(0.1)$ & \\
\hline mcp124-3 & 0.7 & & 139 & 123,6 & $0.7(0.2)$ & & 124 & 124 & $=(0.2)$ & \\
\hline mcp124-4 & 0.7 & & 124 & 124 & $=(0.3)$ & & 124 & 124 & $=(0.3)$ & \\
\hline mcp250-1 & 4.8 & & 268 & $217,7,3$ & $4.1(0.3)$ & & 401 & $176,58,7$ & $4.4(0.3)$ & \\
\hline mcp250-2 & 4.6 & & 336 & $235,12,5$ & $5.7(0.6)$ & & 327 & $237,12,5$ & $5.5(0.6)$ & \\
\hline mcp 250-3 & 4.7 & & 444 & $243,11,11$ & $6.8(1.6)$ & & 277 & $247,6,4$ & $4.8(1.7)$ & \\
\hline mcp250-4 & 4.6 & & 305 & 249,11 & $5.2(3.2)$ & & 250 & 250 & $=(3.1)$ & \\
\hline mcp500-1 & 32.8 & & 539 & $405,10,10$ & $29.2(1.9)$ & & 530 & $410,10,10$ & $28.7(2.2)$ & \\
\hline mcp500-2 & 32.9 & & 862 & $439,14,12$ & $70.7(4.5)$ & & 784 & $449,15,9$ & $56.8(4.9)$ & \\
\hline mcp500-3 & 30.9 & & 1175 & $478,18,16$ & $114.0(17.0)$ & & 1019 & $482,16,13$ & $63.8(17.3)$ & \\
\hline $\operatorname{mcp} 500-4$ & 30.9 & & 1823 & $492,20,20$ & $127.2(50.2)$ & & 758 & $498,18,15$ & $35.0(50.5)$ & \\
\hline qpG11 & 810.4 & & 946 & $419,396,5$ & $103.7(8.7)$ & & 1072 & $397,219,216$ & $118.2(9.1)$ & \\
\hline qpG51 & 1735.8 & & 2180 & $950,16,15$ & $1389.6(77.2)$ & & 1838 & $965,16,15$ & $904.0(81.0)$ & \\
\hline ss30 & 52.9 & p6 & 132 & $294,132 \mathrm{~d}$ & $=(0.7)$ & & 132 & $294,132 \mathrm{~d}$ & $=(0.8)$ & \\
\hline theta1 & 0.2 & & 104 & 50 & $=(0.0)$ & & 104 & 50 & $=(0.0)$ & \\
\hline theta 2 & 3.4 & & 498 & 100 & $=(0.3)$ & & 498 & 100 & $=(0.4)$ & \\
\hline theta3 & 22.5 & 6 & 1106 & 150 & $=(1.5)$ & 6 & 1106 & 150 & $=(1.6)$ & \\
\hline theta 4 & 91.0 & 6 & 1949 & 200 & $=(4.6)$ & 6 & 1949 & 200 & $=(4.9)$ & \\
\hline theta 5 & 264.9 & 6 & 3028 & 250 & $=(11.6)$ & 6 & 3028 & 250 & $=(11.6)$ & \\
\hline theta 6 & 659.9 & 6 & 4375 & 300 & $=(24.4)$ & 6 & 4375 & 300 & $=(25.1)$ & \\
\hline thetaG11 & 684.4 & & 2743 & $362,330,145$ & $508.2(20.5)$ & & 2572 & 417,402 & $501.4(28.9)$ & \\
\hline thetaG51 & 11120.2 & 3 p5 & 7210 & 1000,25 & $*(754.4)$ & & 6910 & 1001 & $=(776.0)$ & \\
\hline
\end{tabular}

which provides an enormous shortening of time for sparse SDPs, that can be 10 100 times faster in some cases, even having the risk to face bad problems, that can take 2 times more to solve in most of cases.

Here we omitted the discussion on used memory, but its advantage is clear in general when the computational time is good as well [16].

We have a general impression that the performance of "conversion-fe" is better than "conversion" in the worse case scenarios, when solving the original problem is slightly faster, for all the numerical experiments we completed. A minor remark is that "conversion-fe" 
Table 10: Numerical results on SDPLIB problems for SDPT3 3.02 at computer A.

\begin{tabular}{|c|c|c|c|c|c|c|c|c|c|}
\hline \multirow[b]{2}{*}{ problem } & standard & \multicolumn{4}{|c|}{ conversion } & \multicolumn{4}{|c|}{ conversion-fe } \\
\hline & time $(\mathrm{s}) \mathrm{rg}$ fea & $m_{+}$ & $n_{\max }$ & time $(\mathrm{s})$ & $\mathrm{rg}$ fea & $m_{+}$ & $n_{\max }$ & time $(\mathrm{s})$ & rg fea \\
\hline $\operatorname{arch} 0$ & $22.66 \mathrm{p} 5$ & 174 & $161,174 \mathrm{~d}$ & $=(0.4)$ & & 174 & $161,174 \mathrm{~d}$ & $=(0.5)$ & \\
\hline $\operatorname{arch} 2$ & $21.56 \mathrm{p} 6$ & 174 & $161,174 \mathrm{~d}$ & $=(0.4)$ & & 174 & $161,174 \mathrm{~d}$ & $=(0.5)$ & \\
\hline $\operatorname{arch} 4$ & 21.8 & 174 & $161,174 \mathrm{~d}$ & $=(0.4)$ & & 174 & $161,174 d$ & $=(0.5)$ & \\
\hline $\operatorname{arch} 8$ & 20.8 & 174 & $161,174 \mathrm{~d}$ & $=(0.4)$ & & 174 & $161,174 \mathrm{~d}$ & $=(0.5)$ & \\
\hline equalG11 & $\begin{array}{lll}234.2 & 6 & \mathrm{p} 6\end{array}$ & 2511 & $203,202,202$ & $286.8(6.8)$ & $5 \mathrm{p} 4$ & 1143 & $410,218,209$ & $103.6(12.2)$ & $5 \mathrm{p} 5$ \\
\hline equalG51 & 861.8 & 2682 & $998,52,29$ & $1425.7(679.5)$ & $5 \mathrm{p} 5$ & 1407 & 1000,29 & $1103.2(681.0)$ & p6 \\
\hline gpp100 & 3.1 & 101 & 100 & $=(0.2)$ & & 101 & 100 & $=(0.2)$ & \\
\hline gpp124-1 & 5.4 & 161 & 122,10 & $6.5(0.2)$ & $5 \mathrm{p} 5$ & 125 & 124 & $=(0.2)$ & \\
\hline gpp124-2 & 5.7 & 146 & 123,7 & $6.0(0.4)$ & & 146 & 123,7 & $6.0(0.4)$ & \\
\hline gpp124-3 & 4.3 & 125 & 124 & $=(0.5)$ & & 125 & 124 & $=(0.5)$ & \\
\hline gpp124-4 & 5.0 & 125 & 124 & $=(0.7)$ & & 125 & 124 & $=(0.7)$ & \\
\hline gpp250-1 & $15.85 \mathrm{p} 5$ & 282 & $248,7,5$ & $21.1(1.7)$ & $6 \mathrm{p} 5$ & 251 & 250 & $=(1.8)$ & \\
\hline gpp250-2 & 16.8 & 251 & 250 & $=(3.3)$ & & 251 & 250 & $=(3.4)$ & \\
\hline gpp250-3 & 17.7 & 251 & 250 & $=(4.9)$ & & 251 & 250 & $=(5.0)$ & \\
\hline gрp250-4 & 14.9 & 251 & 250 & $=(6.9)$ & & 251 & 250 & $=(6.9)$ & \\
\hline gpp500-1 & 108.26 & 908 & $492,17,13$ & $163.3(16.1)$ & $5 \mathrm{p} 4$ & 537 & 499,9 & $120.6(16.7)$ & 5 \\
\hline gpp500-2 & 102.06 & 1312 & $495,30,26$ & $172.4(41.1)$ & $5 \mathrm{p} 6$ & 501 & 500 & $=(37.3)$ & \\
\hline gpp500-3 & $122.76 \mathrm{p} 6$ & 1356 & $496,28,27$ & $198.0(56.6)$ & $\mathrm{p} 4$ & 654 & 499,18 & $129.9(57.1)$ & $5 \mathrm{p} 6$ \\
\hline gpp500-4 & $119.56 \mathrm{p} 6$ & 501 & 500 & $=(88.5)$ & $6 \mathrm{p} 6$ & 501 & 500 & $=(88.7)$ & \\
\hline $\operatorname{maxG11}$ & 178.9 & 2228 & $178,178,178$ & $162.1(5.3)$ & & 1208 & $216,216,208$ & $62.2(8.2)$ & \\
\hline $\max G 32$ & 2017.6 & 5310 & $528,526,513$ & $1908.2(161.1)$ & & 2000 & 2000 & $=(357.0)$ & \\
\hline $\max \mathrm{G} 51$ & 531.5 & 2501 & $937,16,15$ & $658.5(82.3)$ & & 1677 & $971,15,15$ & $550.7(88.7)$ & \\
\hline mcp100 & 3.0 & 141 & $90,7,5$ & $3.6(0.1)$ & & 107 & $95,5,3$ & $3.3(0.1)$ & \\
\hline mcp124-1 & 3.3 & 167 & $85,18,11$ & $3.2(0.1)$ & & 184 & $60,48,13$ & $3.4(0.1)$ & \\
\hline mcp124-2 & 3.6 & 157 & $118,5,5$ & $4.0(0.2)$ & & 144 & $120,5,4$ & $4.3(0.2)$ & \\
\hline mсp124-3 & 4.2 & 188 & $121,8,7$ & $5.0(0.2)$ & & 124 & 124 & $=(0.3)$ & \\
\hline mсp124-4 & 4.6 & 124 & 124 & $=(0.4)$ & & 124 & 124 & $=(0.4)$ & \\
\hline mср250-1 & 9.3 & 436 & $170,56,11$ & $14.7(0.4)$ & & 401 & $176,58,7$ & $13.8(0.5)$ & \\
\hline mср250-2 & 10.4 & 342 & $233,12,5$ & $11.3(0.9)$ & & 327 & $237,12,5$ & $11.4(1.1)$ & \\
\hline mср250-3 & 12.9 & 472 & $242,11,11$ & $17.1(1.9)$ & & 277 & $247,6,4$ & $13.5(2.1)$ & \\
\hline mср250-4 & 13.4 & 736 & $246,20,17$ & $22.9(3.5)$ & & 250 & 250 & $=(3.4)$ & \\
\hline mср500-1 & 51.5 & 582 & $388,11,10$ & $35.2(3.3)$ & & 530 & $410,10,10$ & $37.3(4.4)$ & \\
\hline mср500-2 & 64.3 & 987 & $430,15,14$ & $71.3(6.3)$ & & 784 & $449,15,9$ & $64.9(7.3)$ & \\
\hline mср500-3 & 81.6 & 1566 & $467,18,18$ & $142.4(17.2)$ & & 1019 & $482,16,13$ & $96.8(18.2)$ & \\
\hline mср500-4 & 83.9 & 2800 & $486,34,20$ & $370.0(46.1)$ & & 758 & $498,18,15$ & $89.7(46.8)$ & \\
\hline qpG11 & 1835.3 & 1364 & $218,208,204$ & $84.4(10.6)$ & & 1072 & $397,219,216$ & $79.7(16.0)$ & \\
\hline qpG51 & 3876.9 & 2472 & $937,16,15$ & $652.9(87.6)$ & & 1838 & $965,16,15$ & $527.1(99.9)$ & \\
\hline ss30 & $\begin{array}{lll}50.0 & 6 & \mathrm{p} 6 \\
\end{array}$ & 132 & $294,132 \mathrm{~d}$ & $=(1.1)$ & & 132 & $294,132 \mathrm{~d}$ & $=(1.3)$ & \\
\hline theta1 & 1.25 & 104 & 50 & $=(0.0)$ & & 104 & 50 & $=(0.0)$ & \\
\hline theta2 & $9.45 \mathrm{p} 5$ & 498 & 100 & $=(0.5)$ & & 498 & 100 & $=(0.5)$ & \\
\hline theta3 & $50.4 \quad 4 \mathrm{p} 4$ & 1106 & 150 & $=(2.1)$ & & 1106 & 150 & $=(2.3)$ & \\
\hline theta4 & $183.26 \mathrm{p} 5$ & 1949 & 200 & $=(6.2)$ & & 1949 & 200 & $=(6.7)$ & \\
\hline theta 5 & $579.2 \quad 6 \quad \mathrm{p} 5$ & 3028 & 250 & $=(15.0)$ & & 3028 & 250 & $=(15.7)$ & \\
\hline theta6 & $1562.55 \mathrm{p} 5$ & 4375 & 300 & $=(31.1)$ & & 4375 & 300 & $=(32.2)$ & \\
\hline thetaG11 & 1426.1 & 2914 & $224,224,211$ & $1579.4(26.0)$ & $6 \mathrm{p} 5$ & 2572 & 417,402 & $1106.7(51.1)$ & p6 \\
\hline thetaG51 & $*$ & 7210 & 1000,25 & $*(817.8)$ & & 6910 & 1001 & $*(856.0)$ & \\
\hline
\end{tabular}

produces in general similar SDPs in terms of sizes independent of computers and solvers which indicates that "conversion-fe" relies on how we define the flop estimation function.

It also remains the difficult question if it is possible to obtain homogeneous matrix sizes for the converted SDPs (see columns $n_{\max }$ ).

As proposed in Introduction, the conversion method should be considered as a first step to include preprocessing in SDP solvers. A very further project considers incorporating the conversion method in SDPA [25] and SDPARA [26] together with the completion method 
Table 11: Numerical results on SDPLIB problems for SDPT3 3.02 at computer B.

\begin{tabular}{|c|c|c|c|c|c|c|c|c|c|}
\hline \multirow[b]{2}{*}{ problem } & standard & \multicolumn{4}{|c|}{ conversion } & \multicolumn{4}{|c|}{ conversion-fe } \\
\hline & time $(\mathrm{s}) \mathrm{rg}$ fea & $m_{+}$ & $n_{\max }$ & time $(\mathrm{s})$ & rg fea & $m_{+}$ & $n_{\max }$ & time $(\mathrm{s})$ & $\mathrm{rg}$ fea \\
\hline $\operatorname{arch0}$ & $13.16 \mathrm{p} 6$ & 174 & $161,174 d$ & $=(0.2)$ & & 174 & $161,174 \mathrm{~d}$ & $=(0.3)$ & \\
\hline $\operatorname{arch} 2$ & $12.96 \mathrm{p} 6$ & 174 & $161,174 d$ & $=(0.2)$ & & 405 & $100,82,174 \mathrm{~d}$ & $10.6(0.3)$ & $4 \mathrm{p} 5$ \\
\hline $\operatorname{arch} 4$ & 13.1 & 174 & $161,174 \mathrm{~d}$ & $=(0.2)$ & & 405 & $100,82,174 \mathrm{~d}$ & $10.6(0.3)$ & $5 \mathrm{p} 6$ \\
\hline $\operatorname{arch} 8$ & 12.6 & 174 & $161,174 \mathrm{~d}$ & $=(0.2)$ & & 405 & $100,82,174 d$ & $11.1(0.3)$ & $1 \mathrm{p} 4$ \\
\hline equalG11 & 132.26 & 1979 & $214,213,205$ & $161.0(3.8)$ & $5 \mathrm{p} 4$ & 972 & 410,409 & $77.3(8.7)$ & $6 \mathrm{p} 5$ \\
\hline equalG51 & 477.9 & 2682 & $998,52,29$ & $750.1(762.4)$ & $5 \mathrm{p} 6$ & 1407 & 1000,29 & $589.8(762.6)$ & $6 \mathrm{p} 5$ \\
\hline gpp100 & 2.0 & 101 & 100 & $=(0.2)$ & & 101 & 100 & $=(0.2)$ & \\
\hline gpp124-1 & $3.06 \mathrm{p} 6$ & 161 & 122,10 & $4.1(0.2)$ & $5 \mathrm{p} 4$ & 125 & 124 & $=(0.2)$ & \\
\hline gpp124-2 & $3.46 \mathrm{p} 6$ & 146 & 123,7 & $3.6(0.3)$ & 6 & 146 & 123,7 & $3.6(0.3)$ & p6 \\
\hline gpp124-3 & 2.7 & 125 & 124 & $=(0.4)$ & & 125 & 124 & $=(0.4)$ & \\
\hline gpp124-4 & 3.1 & 125 & 124 & $=(0.6)$ & & 125 & 124 & $=(0.6)$ & \\
\hline gpp250-1 & $12.45 \mathrm{p} 5$ & 282 & $248,7,5$ & $16.6(1.3)$ & $6 \mathrm{p} 6$ & 251 & 250 & $=(1.4)$ & \\
\hline gpp250-2 & $12.36 \mathrm{p} 6$ & 251 & 250 & $=(3.1)$ & & 251 & 250 & $=(3.2)$ & \\
\hline gpp250-3 & $13.76 \mathrm{p} 6$ & 251 & 250 & $=(4.9)$ & & 251 & 250 & $=(5.0)$ & \\
\hline gpp250-4 & 11.2 & 251 & 250 & $=(7.0)$ & & 251 & 250 & $=(7.1)$ & \\
\hline gpp500-1 & $64.05 \mathrm{p} 5$ & 908 & $492,17,13$ & $97.0(15.0)$ & 4 & 537 & 499,9 & $80.5(15.2)$ & $6 \mathrm{p} 6$ \\
\hline gpp500-2 & 68.5 & 1312 & $495,30,26$ & $105.5(41.7)$ & 5 p5 & 501 & 500 & $=(39.2)$ & \\
\hline gpp500-3 & $71.16 \mathrm{p} 6$ & 1005 & $498,27,18$ & $97.3(61.9)$ & 5 p5 & 654 & 499,18 & $84.7(62.0)$ & $5 \mathrm{p} 5$ \\
\hline gpp500-4 & 78.4 & 501 & 500 & $=(98.9)$ & & 501 & 500 & $=(98.8)$ & \\
\hline $\operatorname{maxG11}$ & 98.5 & 1808 & $195,192,190$ & $85.4(3.2)$ & & 1072 & $408,216,208$ & $48.9(5.8)$ & \\
\hline $\operatorname{maxG} 32$ & 1054.3 & 5310 & $528,526,513$ & $1034.0(91.7)$ & & 2000 & 2000 & $=(182.2)$ & \\
\hline $\max G 51$ & 287.7 & 2414 & $941,16,15$ & $347.5(72.2)$ & & 1677 & $971,15,15$ & $306.8(75.7)$ & \\
\hline mcp100 & 2.1 & 138 & $91,7,5$ & $2.3(0.0)$ & & 107 & $95,5,3$ & $2.1(0.1)$ & \\
\hline mcp124-1 & 2.2 & 145 & $97,13,5$ & $2.3(0.0)$ & & 184 & $60,48,13$ & $2.1(0.1)$ & \\
\hline mсp124-2 & 2.3 & 163 & $117,5,5$ & $2.4(0.1)$ & & 144 & $120,5,4$ & $2.7(0.1)$ & \\
\hline mсp124-3 & 2.7 & 188 & $121,8,7$ & $3.1(0.2)$ & & 124 & 124 & $=(0.2)$ & \\
\hline mср124-4 & 3.0 & 124 & 124 & $=(0.3)$ & & 124 & 124 & $=(0.3)$ & \\
\hline mср250-1 & 8.1 & 305 & $209,9,7$ & $6.6(0.3)$ & & 401 & $176,58,7$ & $8.5(0.3)$ & \\
\hline mcp250-2 & 8.8 & 420 & $228,17,12$ & $9.1(0.5)$ & & 327 & $237,12,5$ & $8.8(0.6)$ & \\
\hline mсp250-3 & 11.6 & 597 & $241,19,11$ & $13.6(1.6)$ & & 277 & $247,6,4$ & $10.7(1.7)$ & \\
\hline mcp250-4 & 12.0 & 495 & $248,20,11$ & $12.5(3.2)$ & & 250 & 250 & $=(3.1)$ & \\
\hline mcp500-1 & 35.7 & 564 & $395,11,10$ & $22.9(1.7)$ & & 530 & $410,10,10$ & $24.7(2.2)$ & \\
\hline mср500-2 & 45.7 & 903 & $435,14,12$ & $42.0(4.5)$ & & 784 & $449,15,9$ & $41.5(4.9)$ & \\
\hline mср500-3 & 59.1 & 1455 & $471,18,18$ & $80.3(16.9)$ & & 1019 & $482,16,13$ & $60.6(17.3)$ & \\
\hline mcp500-4 & 60.4 & 2800 & $486,34,20$ & $209.5(50.0)$ & & 758 & $498,18,15$ & $58.5(50.6)$ & \\
\hline qpG11 & 966.6 & 1354 & $219,208,204$ & $56.5(6.0)$ & & 1072 & $397,219,216$ & $52.8(9.1)$ & \\
\hline qpG51 & 1901.4 & 2372 & $941,16,15$ & $342.5(77.1)$ & & 1838 & $965,16,15$ & $292.5(80.6)$ & \\
\hline ss30 & $\begin{array}{lll}33.5 & 6 & \mathrm{p} 6\end{array}$ & 132 & $294,132 \mathrm{~d}$ & $=(0.7)$ & & 132 & $294,132 \mathrm{~d}$ & $=(0.8)$ & \\
\hline theta1 & 0.85 & 104 & 50 & $=(0.0)$ & & 104 & 50 & $=(0.0)$ & \\
\hline theta2 & $5.66 \mathrm{p} 6$ & 498 & 100 & $=(0.3)$ & & 498 & 100 & $=(0.4)$ & \\
\hline theta3 & $29.45 \mathrm{p} 6$ & 1106 & 150 & $=(1.5)$ & & 1106 & 150 & $=(1.6)$ & \\
\hline theta4 & $107.75 \mathrm{p} 5$ & 1949 & 200 & $=(4.6)$ & & 1949 & 200 & $=(4.8)$ & \\
\hline theta5 & $333.0 \quad 6 \mathrm{p} 6$ & 3028 & 250 & $=(11.7)$ & & 3028 & 250 & $=(11.9)$ & \\
\hline theta6 & $895.35 \mathrm{p} 5$ & 4375 & 300 & $=(24.4)$ & & 4375 & 300 & $=(25.2)$ & \\
\hline thetaG11 & 823.3 & 2743 & $389,224,224$ & $626.2(15.8)$ & 6 & 2572 & 417,402 & $593.4(29.0)$ & p6 \\
\hline thetaG51 & $*$ & 7210 & 1000,25 & $*(756.4)$ & & 6910 & 1001 & $*(776.1)$ & \\
\hline
\end{tabular}

$[8,16,17]$, and further develop theoretical and practical algorithms to explore sparsity and eliminate degeneracies.

\section{Acknowledgment}

The second author is grateful for his funding from the National Science Foundation (NSF) via Grant No. ITR-DMS 0113852, and also to Douglas N. Arnold and Masakazu Kojima 
Table 12: Sizes of structural optimization problems.

\begin{tabular}{l|r|r|r|r}
\hline problem & \multicolumn{1}{|c|}{$m$} & \multicolumn{1}{|c|}{$n$} & aggregate (\%) & extended (\%) \\
\hline buck1 & 36 & $24,25,36 \mathrm{~d}$ & 35.65 & 42.44 \\
buck2 & 144 & $96,97,144 \mathrm{~d}$ & 10.60 & 13.94 \\
buck3 & 544 & $320,321,544 \mathrm{~d}$ & 3.67 & 7.40 \\
buck4 & 1200 & $672,673,1200 \mathrm{~d}$ & 1.85 & 5.14 \\
buck5 & 3280 & $1760,1761,3280 \mathrm{~d}$ & 0.74 & 2.98 \\
\hline shmup1 & 16 & $41,40,32 \mathrm{~d}$ & 34.80 & 40.48 \\
shmup2 & 200 & $441,440,400 \mathrm{~d}$ & 4.03 & 10.26 \\
shmup3 & 420 & $901,900,840 \mathrm{~d}$ & 2.03 & 6.24 \\
shmup4 & 800 & $1681,1680,1600 \mathrm{~d}$ & 1.11 & 4.27 \\
shmup5 & 1800 & $3721,3720,3600 \mathrm{~d}$ & 0.51 & 2.49 \\
\hline trto1 & 36 & $25,36 \mathrm{~d}$ & 39.79 & 42.81 \\
trto2 & 144 & $97,144 \mathrm{~d}$ & 12.17 & 14.12 \\
trto3 & 544 & $321,544 \mathrm{~d}$ & 4.22 & 7.50 \\
trto4 & 1200 & $673,1200 \mathrm{~d}$ & 2.12 & 5.52 \\
trto5 & 3280 & $1761,3280 \mathrm{~d}$ & 0.85 & 3.01 \\
\hline vibra1 & 36 & $24,25,36 \mathrm{~d}$ & 35.65 & 42.44 \\
vibra2 & 144 & $96,97,144 \mathrm{~d}$ & 10.60 & 13.94 \\
vibra3 & 544 & $320,321,544 \mathrm{~d}$ & 3.67 & 7.40 \\
vibra4 & 1200 & $672,673,1200 \mathrm{~d}$ & 1.85 & 5.14 \\
vibra5 & 3280 & $1760,1761,3280 \mathrm{~d}$ & 0.74 & 2.98 \\
\hline
\end{tabular}

from Institute for Mathematics and its Applications and Tokyo Institute of Technology for inviting him as a visitor researcher at their respective institutes making this research possible. In particular, Masakazu Kojima contributed with valuable comments on this paper. The authors would like to thank Shigeru Mase and Makoto Yamashita from Tokyo Institute of Technology for suggesting them of using ANOVA. Finally, they are grateful for the kindness of Takashi Tsuchiya from The Institute of Statistical Mathematics for point out the reference [15].

\section{References}

[1] E.D. Andersen and K.D. Andersen, "Presolving in linear programming," Mathematical Programming, vol. 71, pp. 221-245, 1995.

[2] C. Ashcraft, D. Pierce, D.K. Wah, and J. Wu, "The reference manual for SPOOLES, release 2.2: An object oriented software library for solving sparse linear systems of equations," Boeing Shared Services Group, Seattle, WA, January 1999. Available at http://cm.bell-labs.com/netlib/linalg/spooles. 
Table 13: Numerical results on structural optimization problems for SDPA 6.00 at computer A.

\begin{tabular}{|c|c|c|c|c|c|c|c|c|c|}
\hline \multirow[b]{2}{*}{ problem } & standard & \multicolumn{4}{|c|}{ conversion } & \multicolumn{4}{|c|}{ conversion-fe } \\
\hline & time (s) $\mathrm{rg}$ fea & $m_{+}$ & $n_{\max }$ & time $(\mathrm{s})$ & $\mathrm{rg}$ fea & $m_{+}$ & $n_{\max }$ & time $(\mathrm{s})$ & $\mathrm{rg}$ fea \\
\hline buck1 & 0.2 & 36 & $25,24,36 \mathrm{~d}$ & $=(0.0)$ & & 67 & $22,16,15$ & $0.3(0.0)$ & \\
\hline buck2 & 5.26 & 155 & $96,94,6$ & $3.8(0.2)$ & & 202 & $91,51,51$ & $3.7(0.3)$ & 4 \\
\hline buck3 & $142.65 \mathrm{p} 6$ & 774 & $314,201,131$ & $153.8(6.0)$ & $4 \mathrm{p} 5$ & 722 & $318,180,154$ & $185.6(6.9)$ & $5 \mathrm{p} 4$ \\
\hline buck 4 & 1437.8 & 2580 & $400,369,297$ & $3026.7(34.6)$ & $5 \mathrm{p} 5$ & 2691 & $637,458,235$ & $4004.2(63.7)$ & $4 \mathrm{p} 6$ \\
\hline buck5 & $33373.85 \mathrm{p} 6$ & 7614 & $608,530,459$ & $68446.4(430.4)$ & $2 \mathrm{p} 4$ & 5254 & $1043,976,789$ & $33730.9(732.7)$ & $2 \mathrm{p} 4$ \\
\hline shmup1 & 0.4 & 19 & $40,40,3$ & $0.4(0.1)$ & & 74 & $40,32,18$ & $0.4(0.0)$ & 5 \\
\hline shmup2 & 354.8 & 203 & $440,440,3$ & $288.2(4.4)$ & $5 \mathrm{p} 6$ & 709 & $242,242,220$ & $192.2(4.5)$ & $4 \mathrm{p} 5$ \\
\hline shmup3 & 2620.95 & 885 & $900,480,451$ & $1544.3(29.1)$ & 4 & 885 & $900,480,451$ & $1544.1(33.5)$ & 4 \\
\hline shmup4 & 21598.45 & 2609 & $882,882,840$ & $6155.5(174.8)$ & 3 & 1706 & $1680,882,840$ & $8962.2(216.5)$ & 3 \\
\hline shmup5 & $\mathrm{M}$ & 10171 & $1922,1861,1860$ & M (1874.0) & & 5706 & $1922,1922,1861$ & M (2359.4) & \\
\hline trto1 & 0.1 & 36 & $25,36 \mathrm{~d}$ & $=(0.0)$ & & 57 & $16,15,36 \mathrm{~d}$ & $0.1(0.0)$ & 6 \\
\hline trto2 & 2.16 & 155 & $94,6,2$ & $2.0(0.1)$ & $5 \mathrm{p} 6$ & 165 & $88,15,144 d$ & $1.6(0.2)$ & $5 \mathrm{p} 5$ \\
\hline trto3 & $71.26 \mathrm{p} 6$ & 652 & $183,147,7$ & $59.3(1.7)$ & $5 \mathrm{p} 4$ & 760 & $223,112,7$ & $87.6(3.2)$ & $5 \mathrm{p} 4$ \\
\hline trto4 & $762.75 \mathrm{p} 5$ & 1542 & $392,293,12$ & $798.4(16.5)$ & $4 \mathrm{p} 3$ & 1539 & $405,284,12$ & $764.5(23.1)$ & $4 \mathrm{p} 3$ \\
\hline trto5 & $12036.54 \mathrm{p} 5$ & 4111 & $905,498,406$ & $13814.0(230.0)$ & $3 \mathrm{p} 4$ & 4235 & $934,844,32$ & $14864.7(262.4)$ & $3 \mathrm{p} 4$ \\
\hline vibra1 & 0.2 & 36 & $25,24,36 \mathrm{~d}$ & $=(0.0)$ & & 67 & $22,16,15$ & $0.2(0.0)$ & 6 \\
\hline vibra2 & 5.3 & 155 & $96,94,6$ & $5.0(0.2)$ & & 202 & $91,51,51$ & $3.8(0.3)$ & $5 \mathrm{p} 5$ \\
\hline vibra3 & $170.75 \mathrm{p} 6$ & 774 & $314,201,131$ & $183.4(6.1)$ & $4 \mathrm{p} 5$ & 722 & $318,180,154$ & $169.1(6.8)$ & 4 p5 \\
\hline vibra4 & 1596.95 p6 & 2580 & $400,369,297$ & $2717.5(35.3)$ & 4 p3 & 2691 & $637,458,235$ & $3436.2(63.7)$ & $4 \mathrm{p} 4$ \\
\hline vibra5 & 32946.85 p5 & 7614 & $608,530,459$ & 61118.3 (430.4) & $3 \mathrm{p} 4$ & 5254 & $1043,976,789$ & $29979.9(732.6)$ & $3 \mathrm{p} 4$ \\
\hline
\end{tabular}

Table 14: Numerical results on structural optimization problems for SDPA 6.00 at computer B.

\begin{tabular}{|c|c|c|c|c|c|c|c|c|c|c|}
\hline \multirow[b]{2}{*}{ problem } & \multicolumn{2}{|c|}{ standard } & \multicolumn{4}{|c|}{ conversion } & \multicolumn{4}{|c|}{ conversion-fe } \\
\hline & time $(\mathrm{s})$ & $\mathrm{rg}$ fea & $m_{+}$ & $n_{\max }$ & time $(\mathrm{s})$ & $\mathrm{rg}$ fea & $m_{+}$ & $n_{\max }$ & time $(\mathrm{s})$ & $\mathrm{rg} \mathrm{fe}$ \\
\hline buck1 & 0.1 & & 36 & $25,24,36 \mathrm{~d}$ & $=(0.0)$ & & 46 & $25,22,6$ & $0.1(0.0)$ & \\
\hline buck2 & 2.0 & & 160 & $96,92,9$ & $2.1(0.1)$ & 5 & 166 & $96,51,51$ & $1.9(0.2)$ & 6 \\
\hline buck3 & 76.7 & $5 \mathrm{p} 6$ & 794 & $314,205,125$ & $108.2(3.5)$ & $4 \mathrm{p} 5$ & 686 & $318,317,14$ & $95.6(4.4)$ & $4 \mathrm{p}$ \\
\hline buck4 & 714.4 & & 2286 & $371,346,338$ & 2110.9 (19.9) & $6 \mathrm{p} 5$ & 2391 & $669,637,30$ & $2488.2(30.8)$ & $5 \mathrm{pt}$ \\
\hline buck5 & 16667.3 & $5 \mathrm{p} 6$ & 6692 & $639,609,599$ & $46381.0(367.7)$ & $3 \mathrm{p} 4$ & 5254 & $1043,976,789$ & $24194.2(383.7)$ & $2 \mathrm{p}$ \\
\hline shmup1 & 0.2 & & 19 & $40,40,3$ & $0.2(0.0)$ & & 19 & $40,40,3$ & $0.2(0.0)$ & \\
\hline shmup2 & 148.3 & 5 & 203 & $440,440,3$ & $115.0(2.5)$ & 5 & 456 & $440,242,220$ & $102.5(2.8)$ & 4 \\
\hline shmup3 & 968.1 & 5 & 420 & $901,900,840 \mathrm{~d}$ & $=(17.4)$ & & 885 & $900,480,451$ & $635.2(18.0)$ & 4 \\
\hline shmup4 & 5536.6 & 5 & 2609 & $882,882,840$ & $3151.7(97.5)$ & 3 & 1706 & $1680,882,840$ & 3407.9 (112.1) & 3 \\
\hline shmup5 & M & & 5706 & $1922,1922,1861$ & M (1156.9) & & 5706 & $1922,1922,1861$ & M (1237.3) & \\
\hline trto1 & $\overline{0.0}$ & & 36 & $25,36 \mathrm{~d}$ & $=(0.0)$ & & 36 & $25,36 \mathrm{~d}$ & $=(0.0)$ & \\
\hline trto2 & 1.0 & 6 & 160 & $92,9,2$ & $1.0(0.1)$ & 5 p5 & 181 & $91,13,2$ & $1.2(0.1)$ & $4 \mathrm{p}$ \\
\hline trto3 & 40.4 & $5 \mathrm{p} 6$ & 779 & $313,14,12$ & $77.0(1.7)$ & $3 \mathrm{p} 4$ & 607 & $318,7,7$ & $61.1(1.7)$ & $5 \mathrm{p}$ \\
\hline trto4 & 424.1 & $5 \mathrm{p} 5$ & 1734 & $401,283,12$ & $735.6(12.5)$ & $4 \mathrm{p} 4$ & 1539 & $405,284,12$ & $497.9(13.6)$ & $3 \mathrm{p}$ \\
\hline trto5 & 7281.3 & $4 \mathrm{p} 5$ & 4117 & $725,588,498$ & $9382.1(147.6)$ & 3 p5 & 4235 & $934,844,32$ & $10734.3(133.6)$ & $3 \mathrm{p}$ \\
\hline vibra1 & 0.1 & & 36 & $25,24,36 \mathrm{~d}$ & $=(0.0)$ & & 46 & $25,22,6$ & $0.1(0.0)$ & \\
\hline vibra2 & 2.5 & & 160 & $96,92,9$ & $2.6(0.1)$ & 6 & 166 & $96,51,51$ & $1.8(0.2)$ & $5 \mathrm{pt}$ \\
\hline vibra3 & 91.2 & $5 \mathrm{p} 6$ & 794 & $314,205,125$ & $131.7(3.5)$ & $4 \mathrm{p} 5$ & 686 & $318,317,14$ & $115.2(4.4)$ & $4 \mathrm{p}^{\mathrm{s}}$ \\
\hline vibra4 & 793.4 & $5 \mathrm{p} 6$ & 2286 & $371,346,338$ & $2212.1(19.5)$ & $5 \mathrm{p} 3$ & 2391 & $669,637,30$ & $2334.1(31.1)$ & $4 \mathrm{p}$ \\
\hline vibra5 & 14737.5 & $5 \mathrm{p} 5$ & 6692 & $639,609,599$ & 42047.7 (368.1) & 2 p3 & 5254 & $1043,976,789$ & $22015.9(383.9)$ & $3 \mathrm{p}$ \\
\hline
\end{tabular}

[3] J.R.S. Blair, and B. Peyton, "An introduction to chordal graphs and clique tress," in Graph Theory and Sparse Matrix Computations, A. George, J.R. Gilbert, J.W.H. Liu (Eds.), Springer-Verlag, pp. 1-29, 1993. 
Table 15: Numerical results on structural optimization problems for SDPT3 3.02 at computer A.

\begin{tabular}{|c|c|c|c|c|c|c|c|c|c|}
\hline \multirow[b]{2}{*}{ problem } & standard & \multicolumn{4}{|c|}{ conversion } & \multicolumn{4}{|c|}{ conversion-fe } \\
\hline & time (s) rg fea & $m_{+}$ & $n_{\max }$ & time $(\mathrm{s})$ & $\mathrm{rg}$ fea & $m_{+}$ & $n_{\max }$ & time $(\mathrm{s})$ & $\mathrm{rg}$ fea \\
\hline buck1 & $\begin{array}{lll}2.5 & \mathrm{p} 6\end{array}$ & 36 & $25,24,36 \mathrm{~d}$ & $=(0.0)$ & & 46 & $25,22,6$ & $2.6(0.0)$ & \\
\hline buck2 & 8.66 & 166 & $96,64,38$ & $10.3(0.2)$ & $6 \mathrm{p} 5$ & 166 & $96,51,51$ & $9.7(0.3)$ & $5 \mathrm{p} 6$ \\
\hline buck3 & $123.04 \mathrm{p} 4$ & 1141 & $190,142,140$ & $181.1(4.2)$ & $3 \mathrm{p} 4$ & 722 & $318,180,154$ & $136.2(6.9)$ & $4 \mathrm{p} 4$ \\
\hline buck4 & 780.75 p5 & 3113 & $282,266,220$ & $1325.6(26.6)$ & 3 p3 & 2391 & $669,637,30$ & $1423.0(60.8)$ & 3 p5 \\
\hline buck5 & 9634.43 p5 & 9066 & $450,423,392$ & $\mathrm{M}(381.2)$ & & 5254 & $1043,976,789$ & $9558.8(732.8)$ & 2 p3 \\
\hline shmup1 & $\begin{array}{lll}3.2 & 6 & \mathrm{p} 6\end{array}$ & 19 & $40,40,3$ & $4.2(0.0)$ & 6 & 19 & $40,40,3$ & $3.5(0.1)$ & 6 \\
\hline shmup2 & $280.06 \mathrm{p} 6$ & 709 & $242,242,220$ & $161.8(3.6)$ & 4 p3 & 709 & $242,242,220$ & $162.3(4.5)$ & 4 p3 \\
\hline shmup3 & $1444.85 \mathrm{p} 5$ & 1350 & $480,480,451$ & $821.8(25.7)$ & 5 p3 & 885 & $900,480,451$ & $1193.7(33.5)$ & $5 \mathrm{p} 5$ \\
\hline shmup4 & $8272.6 \quad 6$ p6 & 3512 & $882,840,840$ & $5110.2(170.8)$ & $6 \mathrm{p} 5$ & 1706 & $1680,882,840$ & $6726.0(216.5)$ & $6 \mathrm{p} 5$ \\
\hline shmup5 & $73395.4 \quad 5 \quad$ p5 & 30363 & $1050,1020,990$ & M (1888.7) & & 5706 & $1922,1922,1861$ & $30199.9(2358.1)$ & $5 \mathrm{p} 4$ \\
\hline trto1 & $1.26 \mathrm{p} 6$ & 36 & $25,36 \mathrm{~d}$ & $=(0.0)$ & & 57 & $16,15,36 \mathrm{~d}$ & $1.3(0.0)$ & $5 \mathrm{p} 5$ \\
\hline trto2 & $5.26 \mathrm{p} 6$ & 166 & $64,38,2$ & $6.0(0.1)$ & $4 \mathrm{p} 4$ & 181 & $91,13,2$ & $7.5(0.2)$ & 4 p3 \\
\hline trto3 & $53.55 \mathrm{p} 4$ & 986 & $184,132,18$ & $126.4(2.0)$ & $5 \mathrm{p} 2$ & 607 & $318,7,7$ & $97.1(3.2)$ & 4 p3 \\
\hline trto4 & $414.94 \mathrm{p} 3$ & 1797 & $245,235,227$ & $490.2(15.0)$ & $3 \mathrm{p} 2$ & 1539 & $405,284,12$ & $466.4(23.1)$ & $2 \mathrm{p} 2$ \\
\hline trto5 & $4934.63 \mathrm{p} 4$ & 7128 & $452,390,382$ & $22572.0(164.6)$ & $4 \mathrm{p} 1$ & 4235 & $934,844,32$ & $6129.6(262.4)$ & $1 \mathrm{p} 2$ \\
\hline vibra1 & $1.66 \mathrm{p} 6$ & 36 & $25,24,36 \mathrm{~d}$ & $=(0.0)$ & & 46 & $25,22,6$ & $1.5(0.0)$ & $6 \mathrm{p} 6$ \\
\hline vibra2 & $10.56 \mathrm{p} 6$ & 166 & $96,64,38$ & $11.2(0.2)$ & $5 \mathrm{p} 5$ & 166 & $96,51,51$ & $10.9(0.3)$ & $5 \mathrm{p} 5$ \\
\hline vibra3 & $129.14 \mathrm{p} 4$ & 1141 & $190,142,140$ & $216.3(4.2)$ & $4 \mathrm{p} 3$ & 722 & $318,180,154$ & $164.3(6.8)$ & $5 \mathrm{p} 4$ \\
\hline vibra4 & $888.05 \mathrm{p} 4$ & 3113 & $282,266,220$ & $1613.7(26.6)$ & $0 \mathrm{p} 2$ & 2391 & $669,637,30$ & $1558.7(60.8)$ & $0 \mathrm{p} 2$ \\
\hline vibra5 & $16327.14 \mathrm{p} 4$ & 9066 & $450,423,392$ & M (381.1) & & 5254 & $1043,976,789$ & $*(732.6)$ & \\
\hline
\end{tabular}

Table 16: Numerical results on structural optimization problems for SDPT3 3.02 at computer B.

\begin{tabular}{|c|c|c|c|c|c|c|c|c|c|}
\hline \multirow[b]{2}{*}{ problem } & standard & \multicolumn{4}{|c|}{ conversion } & \multicolumn{4}{|c|}{ conversion-fe } \\
\hline & time $(\mathrm{s}) \mathrm{rg}$ fea & $m_{+}$ & $n_{\max }$ & time $(\mathrm{s})$ & $\mathrm{rg}$ fea & $m_{+}$ & $n_{\max }$ & time $(\mathrm{s})$ & rg fea \\
\hline buck1 & $1.6 \mathrm{p} 6$ & 36 & $25,24,36 \mathrm{~d}$ & $=(0.0)$ & & 46 & $25,22,6$ & $1.7(0.0)$ & \\
\hline buck2 & 5.4 & 166 & $96,74,28$ & $6.2(0.1)$ & $5 \mathrm{p} 5$ & 166 & $96,51,51$ & $6.2(0.2)$ & $6 \mathrm{p} 4$ \\
\hline buck3 & $77.33 \mathrm{p} 5$ & 968 & $184,145,145$ & $91.5(2.4)$ & $3 \mathrm{p} 4$ & 686 & $318,317,14$ & $91.7(4.3)$ & $4 \mathrm{p} 3$ \\
\hline buck4 & $453.65 \mathrm{p} 5$ & 3358 & $283,245,220$ & $1042.4(14.8)$ & 3 p3 & 2391 & $669,637,30$ & $804.7(31.3)$ & $3 \mathrm{p} 5$ \\
\hline buck 5 & $5118.33 \mathrm{p} 5$ & 7762 & $470,459,423$ & M (241.5) & & 5254 & $1043,976,789$ & $5189.5(384.0)$ & $2 \mathrm{p} 3$ \\
\hline shmup1 & $2.06 \mathrm{p} 6$ & 19 & $40,40,3$ & $2.6(0.0)$ & 6 & 19 & $40,40,3$ & $2.2(0.0)$ & 6 \\
\hline shmup2 & $164.36 \mathrm{p} 6$ & 203 & $440,440,3$ & $161.2(2.5)$ & $5 \mathrm{p} 5$ & 456 & $440,242,220$ & $143.6(2.8)$ & \\
\hline shmup3 & $771.65 \mathrm{p} 5$ & 1353 & $480,480,450$ & $514.6(14.7)$ & $5 \mathrm{p} 3$ & 885 & $900,480,451$ & $638.1(18.0)$ & $5 \mathrm{p} 4$ \\
\hline shmup4 & $4334.46 \mathrm{p} 6$ & 3512 & $882,840,840$ & $2803.8(95.6)$ & $6 \mathrm{p} 5$ & 1706 & $1680,882,840$ & $3585.2(112.6)$ & $6 \mathrm{p} 5$ \\
\hline shmup5 & $32807.7 \quad 2$ & 11575 & $1922,1860,1054$ & M (1099.4) & & 5706 & $1922,1922,1861$ & $15617.9(1236.9)$ & $5 \mathrm{p} 4$ \\
\hline trto1 & $\begin{array}{lll}0.8 & 6 & \mathrm{p} 6\end{array}$ & 36 & $25,36 \mathrm{~d}$ & $=(0.0)$ & & 57 & $16,15,36 \mathrm{~d}$ & $0.8(0.0)$ & $5 \mathrm{p} 5$ \\
\hline trto2 & 3.16 & 166 & $74,28,2$ & $4.3(0.1)$ & $5 \mathrm{p} 5$ & 181 & $91,13,2$ & $4.5(0.1)$ & $4 \mathrm{p} 3$ \\
\hline trto3 & $33.55 \mathrm{p} 4$ & 806 & $184,139,14$ & $70.0(1.2)$ & 2 p2 & 607 & $318,7,7$ & $61.0(1.7)$ & $4 \mathrm{p} 3$ \\
\hline trto4 & $234.8 \quad 4 \quad$ p3 & 1863 & $273,225,206$ & $349.2(9.6)$ & $3 \mathrm{p} 2$ & 1539 & $405,284,12$ & $279.3(13.5)$ & $3 \mathrm{p} 2$ \\
\hline trto5 & $2598.43 \mathrm{p} 4$ & 7068 & $444,405,372$ & $8028.3(111.0)$ & $4 \mathrm{p} 2$ & 4235 & $934,844,32$ & $3338.9(134.2)$ & $1 \mathrm{p} 2$ \\
\hline vibra1 & $1.06 \mathrm{p} 6$ & 36 & $25,24,36 \mathrm{~d}$ & $=(0.0)$ & & 46 & $25,22,6$ & $0.9(0.0)$ & $6 \mathrm{p} 6$ \\
\hline vibra2 & $6.16 \mathrm{p} 6$ & 166 & $96,74,28$ & $6.7(0.1)$ & $5 \mathrm{p} 5$ & 166 & $96,51,51$ & $6.4(0.2)$ & $5 \mathrm{p} 5$ \\
\hline vibra3 & $80.8 \quad 4 \quad \mathrm{p} 4$ & 968 & $184,145,145$ & $96.1(2.5)$ & 4 p3 & 686 & $318,317,14$ & $109.2(4.3)$ & $4 \mathrm{p} 4$ \\
\hline vibra4 & $508.75 \mathrm{p} 4$ & 3358 & $283,245,220$ & $1143.6(15.0)$ & $2 \mathrm{p} 3$ & 2391 & $669,637,30$ & $878.2(31.2)$ & $0 \mathrm{p} 2$ \\
\hline vibra5 & $7174.22 \mathrm{p} 5$ & 7762 & $470,459,423$ & M (239.0) & & 5254 & $1043,976,789$ & * (384.3) & \\
\hline
\end{tabular}

[4] B. Borchers, "SDPLIB 1.2, a library of semidefinite programming test problems", Optimization Methods \& Software, vol. 11-12, pp. 683-690, 1999. Available at http://www.nmt.edu/ sdplib/. 
[5] S. Burer, "Semidefinite programming in the space of partial positive semidefinte matrices", SIAM Journal on Optimization, vol. 14, pp. 139-172, 2003.

[6] K. Fujisawa, M. Fukuda, M. Kojima, and K. Nakata, "Numerical evaluations of SDPA (SemiDefinite Programming Algorithm)," in High Performance Optimization, H. Frenk, K. Roos, T. Terlaky, and S. Zhang (Eds.), Kluwer Academic Publishers, pp. 267-301, 2000 .

[7] K. Fujisawa, M. Kojima, and K. Nakata, "Exploiting sparsity in primal-dual interiorpoint methods for semidefinite programming," Mathematical Programming, vol. 79, pp. 235-253, 1997.

[8] M. Fukuda, M. Kojima, K. Murota, and K. Nakata, "Exploiting sparsity in semidefinite programming via matrix completion I: General framework," SIAM Journal on Optimization, vol. 11, pp. 647-674, 2000.

[9] K. Gatermann, and P.A. Parrilo, "Symmetry groups, semidefinite programs, and sums of squares," arXiv:math.AC/0211450, 2002.

[10] R. Grone, C.R. Johnson, E.M. Sá, H. Wolkowicz, "Positive definite completions of partial hermitian matrices," Linear Algebra and its Applications, vol. 58, pp. 109-124, 1984.

[11] G. Karypis, and V. Kumar, "METIS - A software package for partitioning unstructured graphs, partitioning meshes, and computing fill-reducing orderings of sparse matrices, version 4.0 -, " Department of Computer Science/Army HPC Research Center, University of Minneasota, Minneapolis, MN, September 1998. Available at http://wwwusers.cs.umn.edu/ karypis/metis/metis.

[12] M. Kočvara and M. Stingl. Available at http://www2.am.unierlangen.de/ ₹ocvara/pennon/problems.html.

[13] M. Kojima, "Sums of squares relaxations of polynomial semidefinite programs," Research Report B-397, Department of Mathematical and Computing Sciences, Tokyo Institute of Technology, 2-12-1 Oh-Okayama, Meguro-ku, Tokyo 152-8552 Japan, November 2003.

[14] J.B. Lasserre, "Global optimization with polynomials and the problem of moments," SIAM Journal on Optimization, vol. 11, pp. 796-817, 2001.

[15] S.L. Lauritzen, T.P. Speed, and K. Vijayan, "Decomposable graphs and hypergraphs," The Journal of the Australian Mathematical Society, Series A, vol. 36, pp. 12-29, 1984.

[16] K. Nakata, K. Fujisawa, M. Fukuda, M. Kojima, and K. Murota, "Exploiting sparsity in semidefinite programming via matrix completion II: Implementation and numerical results," Mathematical Programming, Series B, vol. 95, pp. 303-327, 2003. 
[17] K. Nakata, M. Yamashita, K. Fujisawa, and M. Kojima, "A parallel primal-dual interior-point method for semidefinite programs using positive definite matrix completion," Research Report B-398, Department of Mathematical and Computing Sciences, Tokyo Institute of Technology, 2-12-1 Oh-Okayama, Meguro-ku, Tokyo 152-8552 Japan, November 2003.

[18] P.A. Parrilo, "Semidefinite programming relaxations for semialgebraic problems," Mathematical Programming, vol. 96, pp. 293-320, 2003.

[19] G.S. Peace, Taguchi Methods: A Hands-on Approach, Addison-Wesley Publishing Company: Reading, Massachusetts, 1993.

[20] H. Sahai, and M.I. Ageel, The Analysis of Variance: Fixed, Random and Mixed Models, Birkhäuser: Boston, 2000.

[21] G. Taguchi, and Y. Yokoyama, Jikken Keikakuho, 2nd ed. (in Japanese), Nihon Kikaku Kyokai: Tokyo, 1987.

[22] K.-C. Toh, "Solving large scale semidefinite programs via an iterative solver on the augmented systems," SIAM Journal on Optimization, vol. 14, pp. 670-698, 2003.

[23] K.-C. Toh, M.J. Todd, and R.H. Tütüncü, "SDPT3 - A MATLAB software package for semidefinite programming, Version 1.3", Optimization Methods \& Software, vol. 11-12, pp. 545-581, 1999. Available at http://www.math.nus.edu.sg/〜mattohkc/sdpt3.html.

[24] J. Whittaker, Graphical Models in Applied Multivariate Statistics, John Wiley \& Sons: New York, 1990.

[25] M. Yamashita, K. Fujisawa, and M. Kojima, "Implementation and evaluation of SDPA 6.0 (SemiDefinite Programming Algorithm 6.0)", Optimization Methods \& Software, vol. 18, pp. 491-505, 2003.

[26] M. Yamashita, K. Fujisawa, and M. Kojima, "SDPARA : SemiDefiniteProgramming Algorithm paRAllel Version", Parallel Computing, vol. 29, pp. 1053-1067, 2003.

[27] Z. Zhao, B.J. Braams, M. Fukuda, M.L. Overton, and J.K. Percus, "The reduced density matrix method for electronic structure calculations and the role of three-index representability conditions," The Journal of Chemical Physics, vol. 120, pp. 2095-2104, 2004. 